\title{
The Dark Matter Time Projection Chamber 4Shooter directional dark matter detector: calibration in a surface laboratory
}

James B. R. Battat ${ }^{\mathrm{j}, *}$, Cosmin Deaconu ${ }^{\mathrm{e}}$, Richard Eggleston ${ }^{\mathrm{i}}$, Peter Fisher ${ }^{\mathrm{e}, \mathrm{f}, \mathrm{g}}$, Pietro Giampa ${ }^{\mathrm{h}}$, Vincent Gregoric $^{\mathrm{b}}$, Shawn Henderson ${ }^{\mathrm{c}}$, Igal Jaegle $^{\mathrm{d}}$, Jay Lawhorn ${ }^{\mathrm{e}}$, Jeremy P. Lopez ${ }^{\mathrm{e}}$, Jocelyn Monroe ${ }^{\mathrm{e}, \mathrm{i}}$, Gabriela Druitt $^{\mathrm{i}}$, Kristen A. Recine ${ }^{\mathrm{b}}$, Adam Strandberg ${ }^{\mathrm{e}}$, Hidefumi Tomita ${ }^{\mathrm{e}}$, Sven Vahsen $^{\mathrm{d}}$, Hermann Wellenstein ${ }^{\mathrm{a}}$

${ }^{a}$ Department of Physics, Brandeis University, Waltham, MA, 02453, USA

${ }^{b}$ Department of Physics, Bryn Mawr College, Bryn Mawr, PA 19010 USA

${ }^{c}$ Department of Physics, Cornell University, Ithaca, NY 14853, USA

${ }^{d}$ Physics Department, University of Hawai'i at Manoa, Honolulu, HI 96822

${ }^{e}$ Physics Department, Massachusetts Institute of Technology, Cambridge, MA 02138, USA

${ }^{f}$ Laboratory for Nuclear Science, Massachusetts Institute of Technology, Cambridge, MA 02139, USA

${ }^{g}$ MIT Kavli Institute for Astrophysics and Space Research, Massachusetts Institute of Technology, Cambridge, MA 02139, USA

${ }^{h}$ Department of Physics, Queen's University, Kingston, Ontario, K7L 3N6, Canada

${ }^{i}$ Department of Physics, Royal Holloway University of London, Egham, Surrey, UK ${ }^{j}$ Physics Department, Wellesley College, Wellesley, MA 02481, USA

\section{Abstract}

The 4Shooter is a prototype dark matter detector built by the Dark Matter Time Projection Chamber (DMTPC) collaboration. The aim of the collaboration is to observe dark matter with directional sensitivity by measuring the recoil directions of nuclei struck by dark matter particles. The 4 Shooter is a single time projection chamber containing $\mathrm{CF}_{4}$ gas, with both optical

\footnotetext{
*Corresponding author

Email address: jbattat@wellesley.edu (James B. R. Battat)
} 
(CCD and photomultiplier tube) and charge readout. This paper describes the 4 Shooter and presents results from the commissioning of the detector in a surface laboratory.

Keywords: Dark matter, WIMP, direct detection, directional detection, DMTPC, CCD, TPC, dark matter wind

\section{Introduction}

2

By now, astrophysical observations provide compelling evidence that over $80 \%$ of the matter content of the universe is non-baryonic [1,2]. Although astrophysical observations constrain the gross anatomy of dark matter, direct detection experiments have not yet produced a definitive detection of dark matter. There are many viable theoretical dark matter candidates [3]. A popular and well-motivated dark matter candidate is the Weakly Interacting Massive Particle (WIMP), and a global effort is underway to detect and characterize the particle properties of WIMPs. This paper presents results from the calibration of the 4Shooter detector, a prototype directional dark matter detector built by the Dark Matter Time Projection Chamber (DMTPC) collaboration.

The field of direct WIMP detection aims to identify the interaction of a dark matter particle with a baryonic target in a detector by measuring WIMP-induced nuclear recoils $[4,5]$. Most of these detectors measure the recoil energy through one or more of ionization, scintillation or thermal energy deposition. A common observable for these detectors is the nuclear recoil energy spectrum (or integrated spectrum in the case of threshold detectors), and the nuclear recoil rate versus time. A challenge in direct detection is 
that the predicted recoil energy spectrum is a featureless falling exponential, which is degenerate with the neutron background-induced energy spectrum. Furthermore, the other main signature, the annual modulation in the event rate, is a few percent effect at realizable thresholds and may be similar to backgrounds that modulate annually $[6,7]$.

The current status of direct WIMP detection is challenging to interpret. At low WIMP mass $\left(\sim 10 \mathrm{GeV} / \mathrm{c}^{2}\right)$, the DAMA/LIBRA and CoGeNT experiments report excesses of events that they attribute to dark matter [8, 9]. Additionally, the three nuclear recoil candidates found by the CDMS silicon search favor a $8.6 \mathrm{GeV} / \mathrm{c}^{2}$ WIMP over a background-only model [10]. Meanwhile, published results from several direct detection experiments $[11,12,13,14]$ exclude some or all of the parameter space of these candidate signals.

Over 25 years ago, nuclear recoil direction was proposed as a more definitive signature for dark matter interactions [15]. The motion of the Earth through the galactic WIMP halo should produce a head-wind of WIMP dark matter and therefore an anisotropy in the direction of nuclear recoils in the galactic frame. This corresponds to a daily directional oscillation of the mean recoil direction in the detector frame. Known backgrounds, on the other hand, are generally isotropic in the galactic frame, so directional detectors can test for anisotropies in the angular recoil spectrum with only a few WIMP events, even in the presence of backgrounds [16, 17, 18, 19, 20]. Because tracking detectors can measure both recoil track length and energy deposition, they can use the charge-to-mass ratio dependency of the stopping power to discriminate signal from backgrounds on an event-by-event 
basis (except in the case of background nuclear recoils, which can be differentiated from signal statistically through the use of directional information). Furthermore, directional detectors could eventually be used for dark matter astrophysics to distinguish between dark matter halo models [21]. For an overview of directional detection see Ref. [22].

The challenge of directional detection is to build a detector with many $\mathrm{kg}$ of target mass while maintaining recoil direction sensitivity. There is a long history of work toward that goal, including gas-based [23] and solid crystal scintillator based detectors $[24,25,26]$. At present, there are six active directional dark matter detection experiments underway worldwide. One group uses nuclear emulsions read out by high-resolution microscopy [27]. The other five make use of diffuse-gas targets in which low-energy nuclear recoil tracks extend $\mathcal{O}(1 \mathrm{~mm})$ and can therefore be reconstructed. These experiments are the Dark Matter Time Projection Chamber (DMTPC) [28], D 3 [29], DRIFT [30], MIMAC [31], and NEWAGE [32]. Of these groups, DMTPC and the latter three have detectors operating underground, and three have set dark matter limits $[28,33,34]$. In addition to these six experiments, there is exploratory work on other technologies including columnar recombination in high pressure (10 bar) xenon gas [35], a biological tracking chamber using strands of DNA anchored to thin gold foils [36], roton anisotropy in liquid helium [37], and continued work on anisotropic photon emission in crystal scintillators [38]. In this work, we describe the DMTPC 4Shooter prototype directional dark matter detector and present basic detector performance measurements. 


\section{4Shooter overview}

The 4Shooter is a Time Projection Chamber (TPC) with both optical (CCD and photomultiplier tube) and charge readout. The CCDs image the TPC amplification plane, and therefore provide a 2D projection of recoil tracks. CCDs provide high spatial resolution with a simple interface (USB cable to a PC) at a low cost per channel. Furthermore, the CCDs couple optically to the detector volume through vacuum viewports and are therefore not in contact with the target gas, reducing sources of outgassing and suppressing alpha backgrounds.

Prior to the 4Shooter, DMTPC demonstrated successful track reconstruction, including vector recoil direction determination (head/tail) with CCDs $[39,40]$. Additionally, a surface run with a 10-liter prototype DMTPC detector (called the 10L) produced a limit on the WIMP-proton spin-dependent interaction that was the strongest limit from a directional detector at the time [28]. The 4Shooter is a factor of two larger in active volume than the 10L and was designed as a platform to test the technologies needed for the next-generation DMTPC detector, a cubic-meter volume detector called DMTPCino [41]. In particular, the 4Shooter design focused on material selection and made use of rigorous cleaning procedures for all detector components. Also, the 4Shooter uses four CCD cameras to make a mosaic image of the full active region of the TPC, as will be done in DMTPCino (in the 10L detector, each CCD imaged a subset of the active region of a single TPC). Based on background studies carried out with the 10L detector, the 4Shooter employs a current-sensitive amplifier for electron recoil rejection [42], and a current monitor on the amplification region power supply for independent 
tagging of spark events in the detector. Finally, the 4Shooter incorporates PMT readout, which along with the charge readout channels can be used to investigate the potential for full 3D tracking and for triggered readout of the CCD cameras.

In this paper, we describe the 4 Shooter detector and readout channels. We also present the results of the surface commissioning of the detector, including the calibration of the CCD and charge readout channels, and measurements of the gas gain and electron diffusion. Forthcoming publications will detail the head-tail reconstruction capability of the 4 Shooter, as well as the algorithms used to identify and reconstruct properties of tracks in the CCD images. Additional detail is provided in Refs. [43, 44].

\section{Choice of detector gas}

An advantage of diffuse-gas TPC detectors is the ability to alter the target gas with little to no modification of the detector hardware. In the past, DMTPC and other groups have experimented with a broad range of detector gases and gas mixtures for dark matter and related applications. For example, the DMTPC group has measured ionization tracks in $\mathrm{Xe}+\mathrm{CF}_{4}$ mixtures [45]. Other directional detection groups use fluorine-rich gases such as $\mathrm{CHF}_{3}$, and the negative-ion drift mixture of $\mathrm{CS}_{2}$ and $\mathrm{CF}_{4}[31,33]$. TPCs with optical readout have also been used with a $\mathrm{He}-\mathrm{CF}_{4}$ mixture to monitor neutron backgrounds at the Double Chooz neutrino experiment [46] and neutrons from fissile material for homeland security applications [47].

The current DMTPC scientific program focuses on the WIMP-proton spin-dependent interaction [48], for which fluorine is a sensitive target [49]. 
The 4Shooter detector uses $\mathrm{CF}_{4}$ gas because of its high fluorine content, and because it has good detector properties, namely high scintillation yield with emission spectrum well-matched to CCD readout [50, 51], and low electron diffusion for a proportional gas [52].

The operating $\mathrm{CF}_{4}$ pressure is typically in the range of 60 to 100 Torr and represents a trade-off between track length and particle stopping power, as well as target mass and stability of detector operation. At higher gas pressure, the larger stopping power enhances the signal-to-noise in a CCD pixel, however the shorter tracks at higher pressure make head-tail reconstruction more challenging. The majority of the commissioning data for the 4Shooter was taken at 60 Torr. Studies have shown $[53,54]$ that for directional detection of $100 \mathrm{GeV} / \mathrm{c}^{2}$ WIMPs, the optimum $\mathrm{CF}_{4}$ operating pressure is $10-30$ Torr (depending on the details of the readout). It would be advantageous to operate the 4 Shooter detector at a lower gas pressure, but we are currently limited by the stability of the amplification region (see Section 8.2).

\section{Vacuum chamber and gas system}

\subsection{Vacuum system}

The active region of the DMTPC 4Shooter detector is housed inside a vacuum chamber (manufactured by Sharon Vacuum in Massachusetts, USA) to contain the $\mathrm{CF}_{4}$ gas and maintain its purity (see Figure 1). This is crucial, as electronegative contaminants such as oxygen capture ionization electrons in the gas and degrade the system gain of the detector. The vacuum chamber interior was electropolished, and metal seals were used where possible to minimize outgassing and permeation into the target gas. 
The vacuum chamber consists of a cylindrical bell jar that mates via a wire seal to a round bottom flange (see Fig. 2). This main seal can be made with either a single-use copper or a reusable elastomer gasket. In the work described here, the elastomer seal was used. The inner diameter of the bell jar is $39.8 \mathrm{~cm}$, and there is $46.4 \mathrm{~cm}$ vertical clearance between the vacuum side of the bell jar lid and the vacuum side of the bottom flange. The main chamber volume is therefere $60 \mathrm{~L}$. The flat top of the bell jar has five ConFlat (CF) optical viewports (four 6" CF for CCD cameras and one 2-3/4" CF for three PMTs, see Section 6). The bottom flange has a 6" CF pump-out port that connects via a 6" to 4-1/2" CF reducer to a pneumatically driven 4-1/2" CF VAT UHV gate valve and then to a Varian V81-M turbo pump with a 4-1/2" CF flange. The turbo is backed by an Edwards XDS-5 dry scroll pump. The chamber pressure is monitored by two pressure gauges attached to the bottom flange. The first gauge is a capacitance manometer, which provides an accurate pressure reading $(0.2 \%)$ independent of gas composition, but only above 0.5 Torr. The second is a combination Bayard-Alpert Pirani gauge, which operates from atmosphere to $10^{-10}$ Torr, but is gas-composition dependent.

\subsection{Gas system}

During standard operation, the chamber is evacuated, typically below $10^{-5}$ Torr, and then back-filled with $\mathrm{CF}_{4}$ gas through a gas-input port on the bottom flange of the chamber. Prior to back-filling, the observed rate of pressure rise is a few millitorr per hour. An MKS 1479A Mass Flow Controller (MFC) regulates the flow rate of the supply gas. Gas fills are done by computer control and can be initiated and monitored through the detector's 

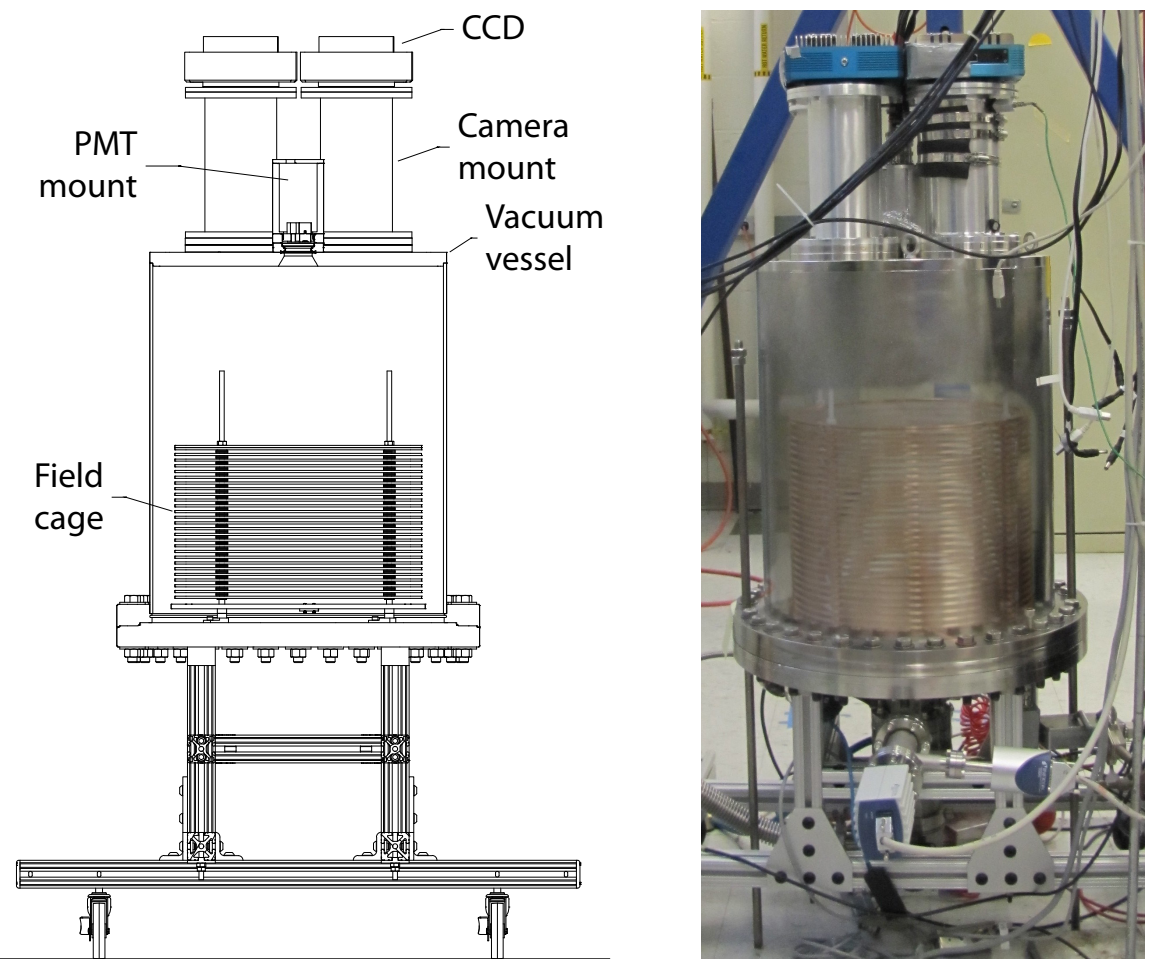

Figure 1: Left: CAD model of the 4Shooter detector, showing two of the four CCD ports on the top of the vessel, as well as the field cage structure inside the vacuum vessel. A single PMT port containing three PMTs is surrounded by the four CCD ports. Right: A composite image of the 4Shooter detector showing the vessel exterior with an overlaid, semi-transparent image of the copper field cage structure contained inside the vessel. 


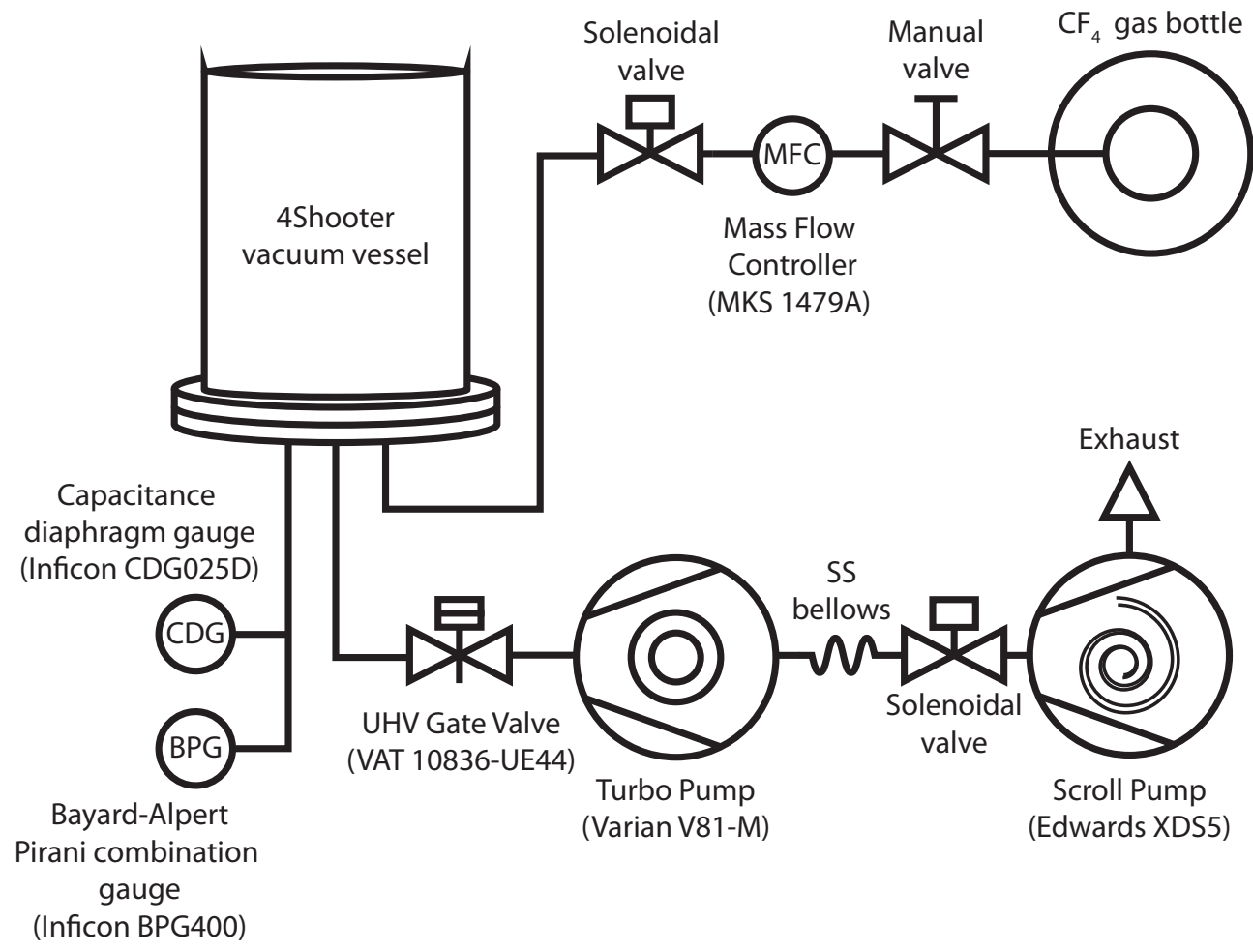

Figure 2: Schematic of the 4Shooter gas and vacuum system.

web interface (see Section 7). When the fill is complete, the electromagnetically actuated valve seals the chamber. An evacuate-and-refill cycle generally lasts $10 \mathrm{~min}$. At present, a gas circulation and purification system is not used, but on a larger detector, such a system may be desirable.

\section{Time Projection Chamber}

The cylindrical TPC is housed inside the vacuum vessel and consists of a drift region and an amplification region (see Figure 3). Ionizing radiation traversing the drift region loses energy through interactions with the surrounding gas. The resulting ionization electrons are driven toward the 
amplification region by a drift field. Once in the amplification region, the electrons experience a large electric field resulting in exponential amplification of the ionization charge, as well as the production of scintillation light from molecular deexcitation. The CCD cameras image the scintillation light through the mesh cathode and ground electrodes. This section describes the drift and amplification regions of the 4 Shooter.

\subsection{Drift region}

The drift region defines the active volume of the detector (see Figure 4). High transparency meshes are used for the drift end-cap electrodes to ensure high optical throughput from the amplification region to the CCDs and PMTs. The top electrode (the cathode) is a woven stainless steel mesh (50 lpi, $30 \mu \mathrm{m}$ wire diameter, $89 \%$ transparency $^{1}$ ) biased at a large negative voltage (generally $-5 \mathrm{kV}$ to minimize electron transverse diffusion in the pressure range $60-100$ Torr). The lower electrode is also a woven stainless steel mesh (100 lpi, $30 \mu \mathrm{m}$ wire diameter, 78\% transparency), grounded through a $20 \Omega$ resistor. Copper field-shaping rings supported by four vertical $1 / 4 "-20$ threaded Delrin rods are connected by $1 \mathrm{M} \Omega$ resistors to establish a uniform electric field defining the drift direction $\hat{z}$. Near the rings, the drift field is non-uniform, and some ionization electrons are therefore captured on the rings (rather than reaching the amplification region). This leads to strong suppression of scintillation light from tracks in the outer $1 \mathrm{~cm}$ of the veto region (see Section 5.2). Each ring is $3 \mathrm{~mm}$ thick with a $30.7 \mathrm{~cm}$ inner diameter

\footnotetext{
${ }^{1}$ Transparency, $T$, refers to the geometric open area, and is given by $T=$ $\left[1-d_{w} *(\mathrm{lpi}-1)\right]^{2}$, where $d_{w}$ is the wire diameter in inches.
} 


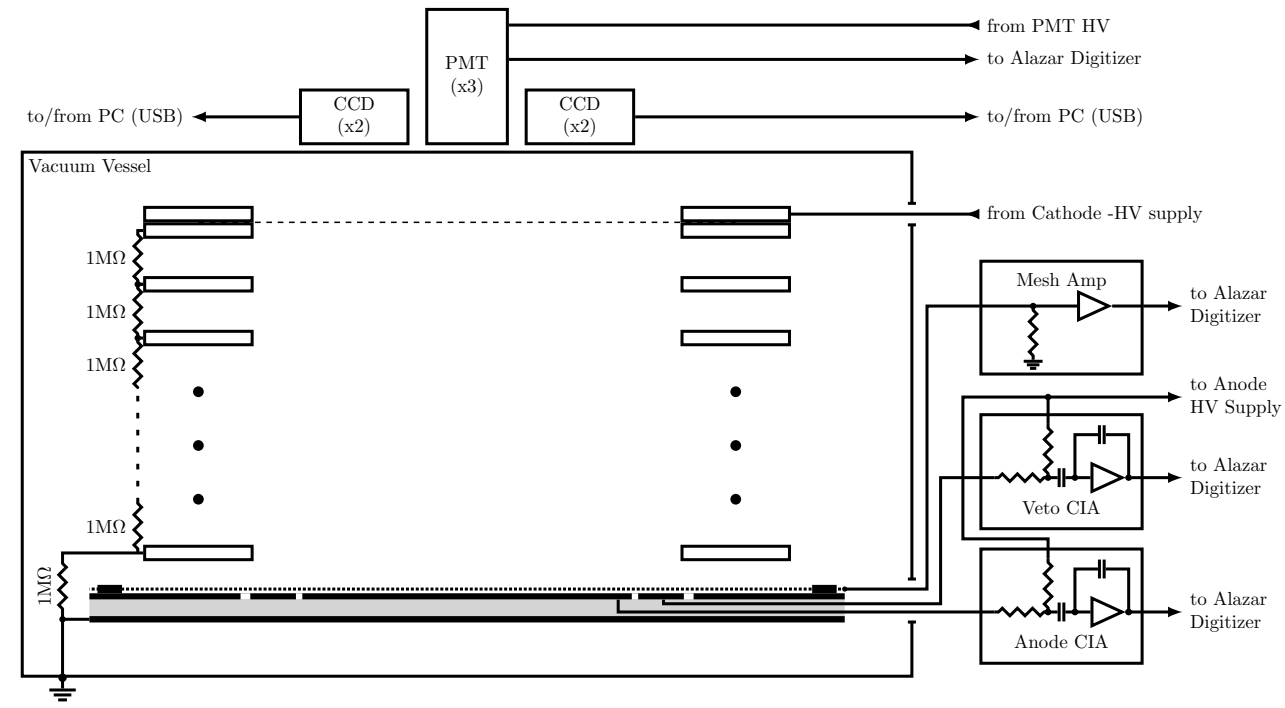

Figure 3: Electrical schematic of the 4Shooter detector including a side-view of the TPC showing several of the field-shaping rings and resistor chain elements. The three electrodes on the top of the anode plate are shown, along with their connections to the three charge readout channels: Mesh Amp, Veto CIA and Anode CIA (CIA stands for Charge Integrating Amplifier). The CCD and PMT readouts are shown atop the vacuum vessel (only two of the four CCDs are shown). Drawing is not to scale.

and a $33.8 \mathrm{~cm}$ outer diameter and is machined from ultra-high purity copper provided by the Aurubis Group. The lowest field-shaping ring is electrically connected to the grounded vacuum chamber via a $1 \mathrm{M} \Omega$ resistor. The cathode mesh is secured under tension to a copper ring using a low-outgassing epoxy (3M DP-460 EG) and then covered by a second ring, both with the same characteristics as the field-shaping rings. In total, there are 28 rings: 26 field-shaping rings and two cathode rings and a total field cage resistance of $27 \mathrm{M} \Omega$. The total drift distance measured $26.7 \pm 0.1 \mathrm{~cm}$.

The field rings are mechanically and electrically separated from each other 


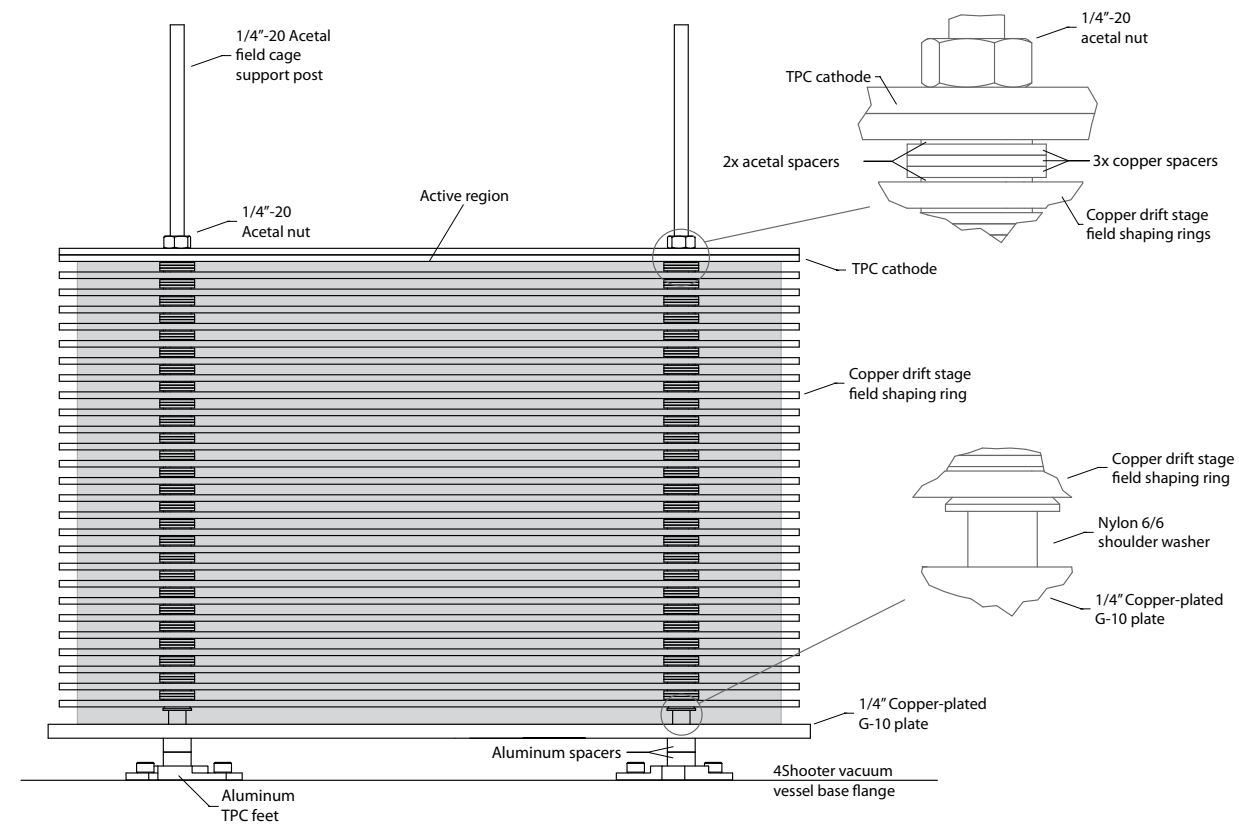

Figure 4: Mechanical drawing of the 4Shooter field cage. The active region of the TPC is indicated by the shaded gray rectangle.

by spacers composed of a stack of three copper washers $(1.65 \pm 0.38 \mathrm{~mm}$ thick $)$ sandwiched between two thin $(0.76 \pm 0.25 \mathrm{~mm})$ Delrin washers. These spacers are primarily composed of copper for material purity. The target size for the spacers is $0.635 \mathrm{~cm}$, and the measured spacer thicknesses range from 0.635 to $0.686 \mathrm{~cm}$. The resistor chain is made of through-hole resistors whose leads tuck under the spacers to make electrical contact with the rings (Fig. 3). The resistors are placed on alternating field cage posts to avoid a tilt in the field cage. Kapton-insulated high-voltage wire connects the cathode to a $30 \mathrm{kV}$ ConFlat high-voltage vacuum feedthrough on the bottom flange of the vessel, which, in turn, is fed by a Bertan 380N NIM high-voltage supply, typically set to $-5 \mathrm{kV}$. 


\subsection{Amplification stage}

The amplification region (see Figure 5) is a custom, monolithic device. It consists of a stainless steel woven mesh epoxied under tension onto a $1 / 4$ " thick copper-clad (on both sides) G-10 plate (the same mesh that serves as the ground electrode for the drift region described above). The mesh-plate gap is defined by 13 non-conductive fused silica capillary tubes ("spacers") of $435 \pm 10 \mu \mathrm{m}$ diameter oriented approximately parallel to each other ${ }^{2}$ on a 1 inch pitch.

The wire pitch of the ground mesh is $257 \mu \mathrm{m}$. The choice of mesh pitch balances spatial resolution (finer mesh), optical transparency (larger gaps), and maximum achievable mesh tension (wire diameter) and therefore the number of required spacers.

Machined channels divide the copper-clad G-10 plate into three electrically isolated regions - the outer, veto and anode electrodes. The mesh is epoxied to the outermost annular region of inner diameter $30.7 \mathrm{~cm}$ and outer diameter flush with the edge of the $34.8 \mathrm{~cm}$ diameter G-10 plate. A second, concentric, annular region (the "veto") of outer diameter $30.7 \mathrm{~cm}$ and inner diameter $29.2 \mathrm{~cm}$ serves as a veto to identify ionization events near the outer radius of the active region (i.e. the electrical signal from this electrode is used for $(x, y)$ fiducialization). Finally, the central $29.2 \mathrm{~cm}$ diameter circular region (the "anode") defines the fiducial region of the detector. A Bertan 375P NIM high-voltage supply biases both the veto and anode electrodes (typically at $670 \mathrm{~V}$ ) to provide Townsend amplification in the narrow gap

\footnotetext{
${ }^{2}$ The ends of the spacers are fixed at precise intervals on the anode plate, but the central portion of the spacers can move.
} 


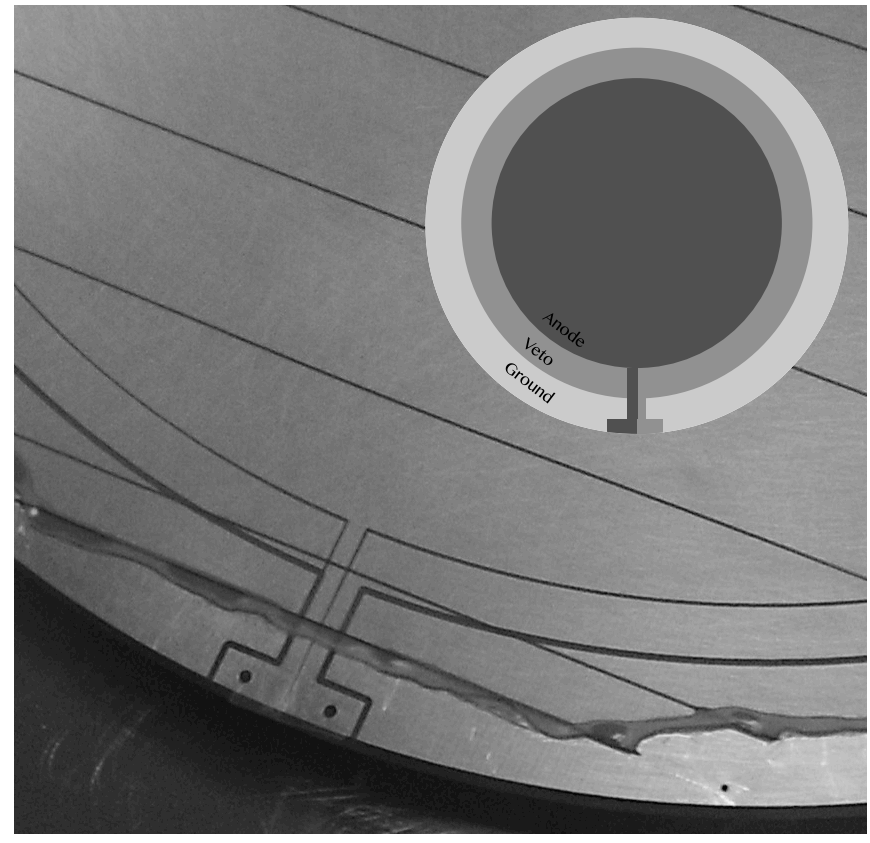

Figure 5: Photograph and schematic drawing of the amplification region. The schematic shows the three distinct electrodes - the central circular anode, surrounded by the annular veto, surrounded by the ground electrode. In addition to these three electrodes, the photograph shows the quartz spacers (parallel lines running from top left to bottom right in the image), and the ground mesh, which is epoxied to the ground electrode. 
(435 $\mu \mathrm{m})$ between the mesh and the anode and veto electrodes.

\subsection{Active volume and target mass}

The active region of the 4 Shooter is defined by the height of the drift region $(26.7 \pm 0.1 \mathrm{~cm})$ and the outer diameter of the central anode region $(29.2 \mathrm{~cm})$, and it has a total volume of $19.8 \mathrm{~L}$. The system gain of the detector near the amplification region spacers is degraded by $20-30 \%$, and in practice additional cuts are made to ignore tracks within $1.3 \mathrm{~mm}$ of spacers. The resulting total fiducial volume is $13.9 \mathrm{~L}$, corresponding to fiducial target masses of fluorine and $\mathrm{CF}_{4}$ at $298 \mathrm{~K}$ and 60 Torr of $3.5 \mathrm{~g}$ and $4.1 \mathrm{~g}$, respectively.

\section{Readout channels}

An ionization event produces two main observable signatures: scintillation light and electron/ion pairs. CCDs image the scintillation light, and PMTs measure the temporal profile of the photon emission. In addition, the integral and temporal profile of the charge signal are measured by charge amplifiers.

\section{1. $C C D s$}

The entire active region of the amplification region is imaged by four CCD cameras, which measure the $2 \mathrm{D}$ projection of the ionization tracks. Each CCD is an Apogee Alta U6 containing a Kodak KAF-1001E frontilluminated CCD. The CCD chips consist of $1024 \times 1024$ pixels, each with $24 \times 24 \mu \mathrm{m}^{2}$ area. Each CCD views the TPC through a multi-element Canon $85 \mathrm{~mm} f / 1.2 \mathrm{SLR}$ lens, and images a $16.4 \times 16.4 \mathrm{~cm}^{2}$ region of the anode. 
Prior to digitization, the CCD pixels are binned $4 \times 4$ on-chip to enhance the signal-to-noise in each digitized bin, and reduce dead-time by shortening readout. In the images shown here, each recorded bin from the CCD images a square region of the anode $0.6416 \mathrm{~mm}$ on a side. The cameras are arranged such that adjacent cameras' fields of view overlap by approximately $1 \mathrm{~cm}$. The CCDs also image the inactive region outside of the field cage. Figure 6 shows the 4-camera mosaic image of an alpha track that traverses the field cage.

The details of the scintillation spectrum of $\mathrm{CF}_{4}$ [50] depend on the gas pressure [55], but in general the spectrum contains two broad emission peaks. One is centered near $300 \mathrm{~nm}$. The other is centered near $625 \mathrm{~nm}$, and is wellmatched to the response of CCD cameras. For example, the Alta U6 cameras have a peak quantum efficiency (QE) of $70 \%$ at $550 \mathrm{~nm}$. The negligible QE of the CCDs below $350 \mathrm{~nm}$ mean that the CCDs are not sensitive to the shortwavelength scintillation photons (200-350 nm), and so standard Kodial glass viewports are used to couple the cameras to the vacuum chamber.

The choice of CCD balances signal to noise and cost for a given field of view. In particular, assuming isotropic photon emission in the amplification region and by making use of the lens-maker's formula, it is possible to express the fraction of scintillation photons $\eta$ that reach the CCD chip as:

$$
\eta=\frac{1}{16}\left(\frac{1}{f / \#}\right)^{2}\left(\frac{1}{1+m}\right)^{2}
$$

where $f / \#$ is the $f$-number of the lens (the ratio of the focal length to the diameter of an equivalent single lens), and $m$ is the demagnification of the optical system (the ratio of the object size to the image size). This expression shows that a fast lens (low $f$-number) and large CCD chip (low $m$ ) 


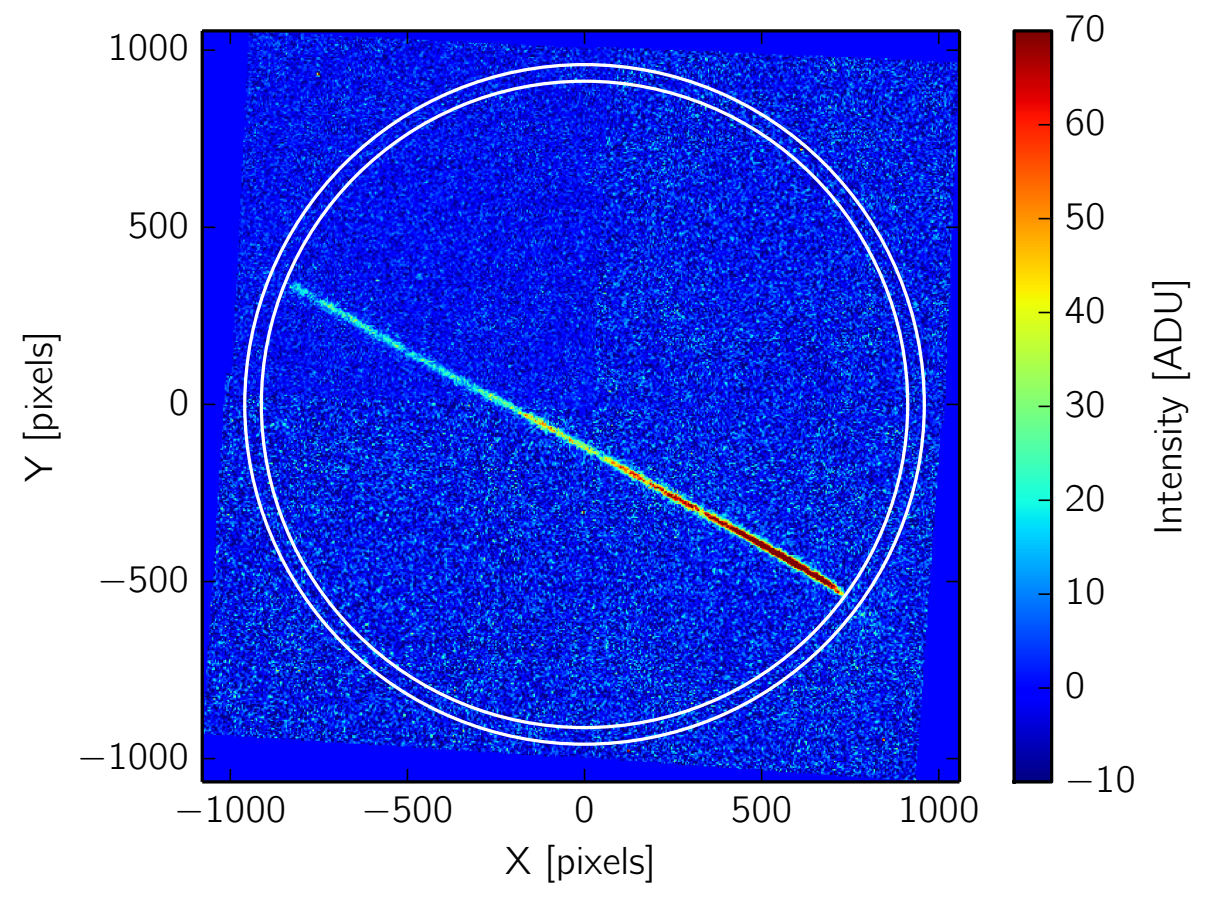

Figure 6: Mosaic CCD image showing an alpha particle traversing the field cage and passing through the fields of view of three CCD cameras. The overlaid circles show the anode-veto boundary (inner circle) and the outer diameter of the veto (outer circle). This alpha likely originated from the decay of an atom near the surface of the copper field-cage ring and terminated on a field cage ring. Regions of suppressed signal along the track are created by the spacers that define the amplification gap. 
are advantageous. It also makes clear the need for a large gas amplification to compensate for the small value of $\eta$ (for the 4Shooter, $f / \#=1.2$ and $m=6.67$, so $\left.\eta=7 \times 10^{-4}\right)$. This expression is purely geometric and does not account for the reflective copper anode, which can boost the photon throughput. Nor does it account for photon losses due to mesh or window or lens transparency or the quantum efficiency of the CCD. The CCD energy calibration, described in Section 8.6, includes all of these factors. The signalto-noise ratio in a CCD pixel could be improved by using back-illuminated CCDs $(\sim 95 \% \mathrm{QE})$ with lower read noise (3 $e^{-}$RMS are readily available now).

Figure 7 shows a nuclear recoil candidate in the 4Shooter detector from an AmBe neutron source exposure. From this data, the following information about the track can be obtained: total ionization energy, total projected track length, stopping $(d E / d x)$ vs. position, track orientation in $2 \mathrm{D}$, track diffusion, and the absolute $(x, y)$ location of tracks, useful for detector fiducialization.

A typical run of the detector consists of 100 dark frames followed by 1000 event exposures. A dark frame is a CCD exposure of the same duration as the event exposures but with the CCD shutter closed. This pattern is repeated for 24 hours. Then the detector is refilled with fresh $\mathrm{CF}_{4}$ gas to ensure gas gain stability (see Section 8.2). At present, no event trigger is implemented, so all images are saved for off-line analysis (witness mode). In typical operation ( 4 cameras, $4 \times 4$ binning, 1-second exposures), the total uncompressed CCD data rate is $0.5 \mathrm{MB} / \mathrm{s}$. We are actively investigating triggered readout using PMT and charge readout information. 


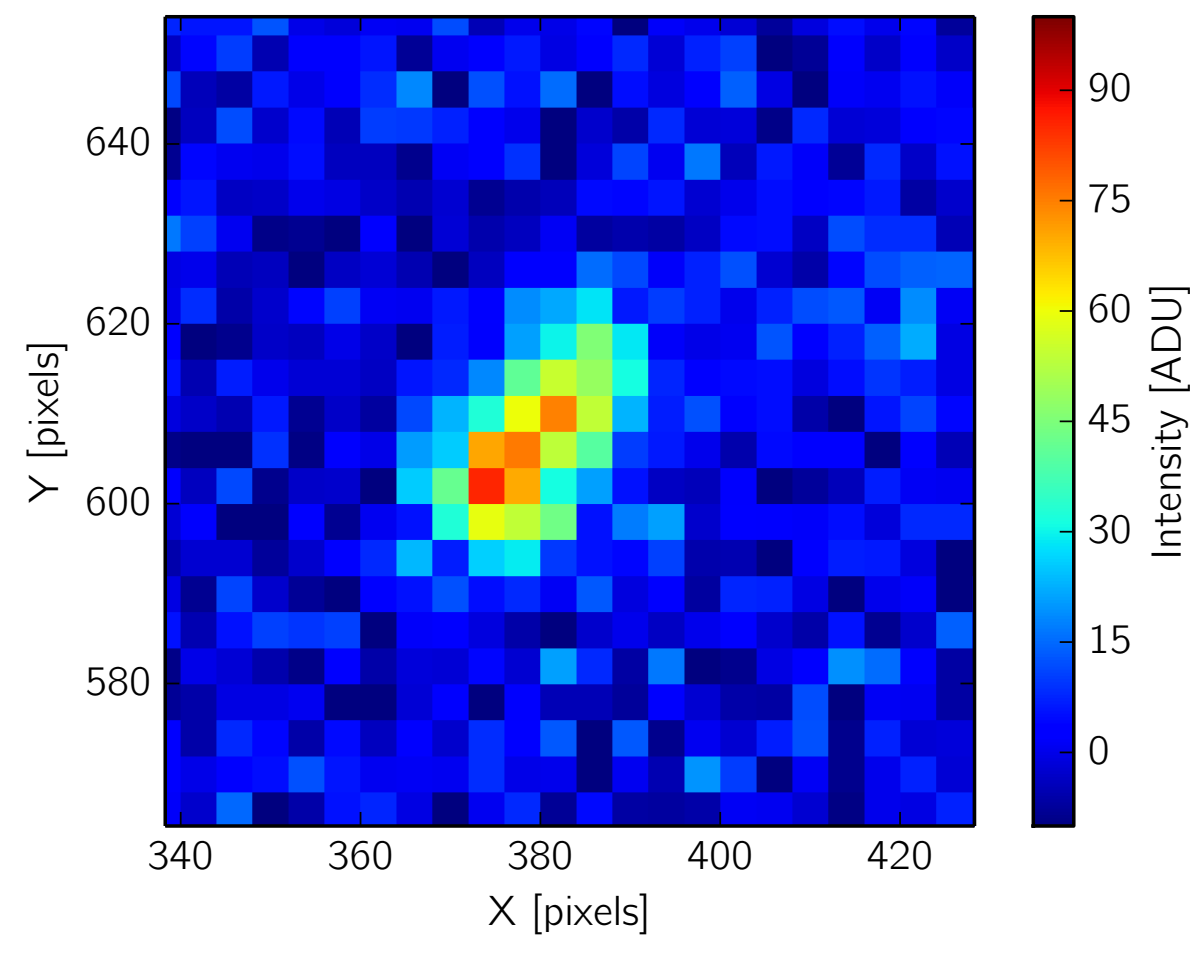

Figure 7: Enlarged view of a high energy $\left(175 \mathrm{keV}_{e e}\right)$ nuclear recoil candidate from an AmBe neutron exposure, imaged by a single CCD. The intensity variation along the track, represented by the color scale, is proportional to $d E / d x$, and indicates that the track travels from bottom left to top right. 


\subsection{Photomultiplier Tubes}

The PMTs provide a complementary measurement of the scintillation light from events in the detector. Like the CCDs, the PMTs sit outside of the active volume and do not contact the detector gas. In the 4Shooter, three face-on 8 mm diameter PMTs (Hamamatsu R7400U-20 with Hamamatsu E5780 bases) are mounted together in the central port of the top flange of the vacuum vessel and couple optically to the chamber through a single 23/4" CF quartz viewport. The PMTs are biased at $-925 \mathrm{~V}$ with Bertan NIM HV supplies, and the output signal is digitized by Alazar ATS860 PCI

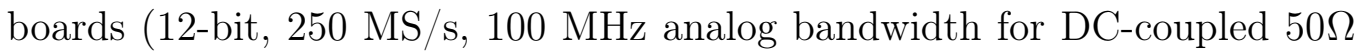
termination). In addition to providing an independent measurement of the energy of an event, the temporal profile of the PMT signal can be used to extract information about the third dimension of the track - tracks with large $\Delta z$ will produce wider pulses in the PMT. This effect was demonstrated with the 4Shooter for high-energy tracks by using an ${ }^{241} \mathrm{Am}$ source and in a similar detector with fixed-length decay products of thermal neutron capture on ${ }^{3} \mathrm{He}$ $\left(n+{ }^{3} \mathrm{He} \longrightarrow p+{ }^{3} \mathrm{H}\right)$ [45]. The bandwidth of the digitizers is not wellmatched to the fast PMT pulses, however, and many PMT waveforms show distortions such as wrong polarity pulses and excess noise. This prevents the full utilization of the PMT channels at low energies, and as a result, the PMT readout is not yet integrated into the data analysis.

\subsection{Charge readout}

The ionization signal in the amplification region is measured in two different ways using three charge amplifiers. All three amplifiers are kept outside 
of the vacuum vessel. The Alazar ATS860 digitizes the charge signals. Figure 8 shows waveforms from the same nuclear recoil candidate event shown in Figure 7.

A current-sensitive amplifier (Route2Electronics HS-AMP-CF) attached to the ground mesh measures the temporal evolution of ionization pulses in the amplification region. DMTPC has previously shown that a pulse-shape analysis of this signal can effectively discriminate between electronic and nuclear recoils [42]. A nuclear recoil event will exhibit a dual-peak structure in the mesh amplifier signal (see Figure 8). The first peak arises from the fast-moving electrons in the amplification region, while the second, broader, peak comes from the slower-moving ions. That work also demonstrated a correlation between $\Delta z$, the vertical extent of a track, and the pulse risetime. Ongoing work explores the possibility of using the amplifier rise-time to measure $\Delta z$ for dark matter induced nuclear recoils.

In addition, two measurements of the total integrated charge after gas amplification are made. First, a Cremat CR-113 charge-sensitive amplifier (nominal gain $1.3 \mathrm{mV} / \mathrm{pC}$ ) mounted on a Cremat CR-150 board integrates the induced charge on the central anode. Second, a Cremat CR-112 chargesensitive amplifier (nominal gain $13 \mathrm{mV} / \mathrm{pC}$ ), also mounted on a CR-150 board, integrates the charge on the veto channel. Both amplifiers have a $300 \Omega$ resistor in series with their inputs to protect against spark discharges in the amplification region. 

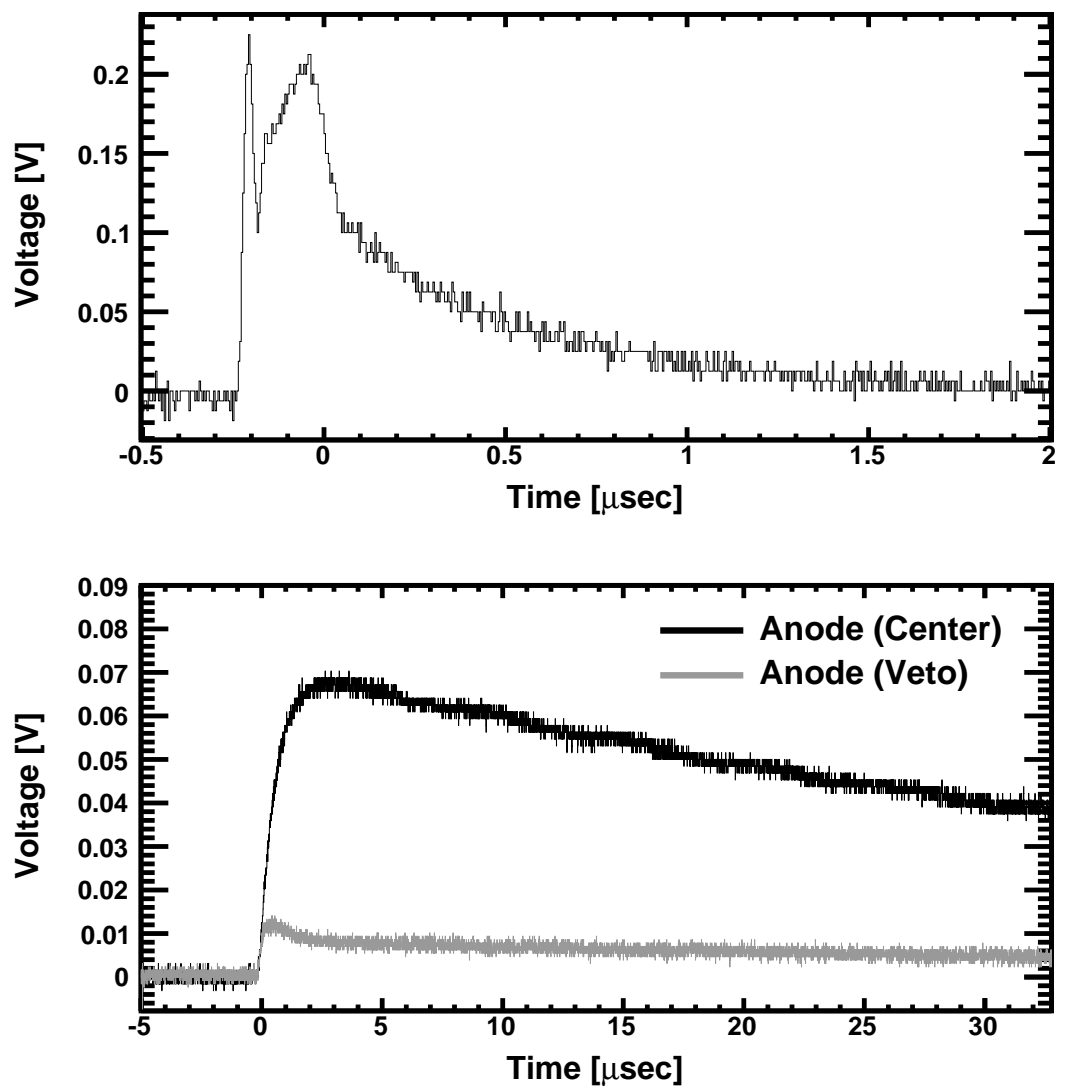

Figure 8: Nuclear recoil candidate waveforms. Top: current-sensitive mesh amplifier. Bottom: central circular anode (upper, black) and annular veto (lower, gray). The top figure shows the separate fast (electron) and slow (ion) peaks in the mesh amplifier signal. 


\section{Hardware control and data acquisition}

The 4Shooter detector was designed to be operated remotely underground. This section briefly describes the Slow Control and DAQ software framework.

\subsection{Control}

The detector control and monitoring scripts (the Slow Control) are a collection of Python, Perl, and $\mathrm{C} / \mathrm{C}++$ code that track and log detector operation parameters to a MySQL database. This code runs on a Linux computer that doubles as a server for a web interface to control and monitor the detector. The TPC voltages and CCD operational parameters (exposure time, number of exposures, binning) can be set and monitored, as can data acquisition parameters such as the waveform digitizer card trigger conditions. The vacuum system can be controlled (valves can be opened and closed), and autonomous detector gas refills to a user-defined pressure can be initiated and monitored. General environmental parameters such as external temperature are also monitored. The measured parameters are recorded at a rate of $1 \mathrm{~Hz}$ and are obtained either by USB or RS-232 connections, or through analog-todigital conversion using a National Instruments NI-6229 multifunction DAQ PCI card.

\subsection{Acquisition}

Data acquisition is handled on a dedicated Linux machine by a suite of $\mathrm{C}++$ code that makes use of the ROOT data analysis framework [56]. Data from the 4Shooter detector is stored in ROOT data files for later off-line 
analysis. The off-line analysis of CCD images and charge and PMT waveforms follows from [57, 43, 44], and will be described in detail in subsequent publications. A Monte Carlo detector simulation of the CCD images has been implemented, with simulated data stored in the same format as the real data, allowing common analyses and intercomparisons of the two.

During acquisition, summary statistics of the incoming data are measured and logged to the database for real-time viewing. For example, dark frames for each run are displayed, as are the mean pixel intensity of the CCD images, as a function of time.

\section{Detector performance}

In this section, we discuss the calibration of the CCD (recoil track length, energy and noise measurements), and charge channels (recoil energy measurement). We also present measurements of the gas gain as a function of gas pressure, anode voltage, and time, as well as the transverse electron diffusion, measured in situ.

\subsection{Length calibration and image mosaic technique}

The calibration of the recoil length scale of the CCD is done in situ when the hardware configuration is altered. This calibration determines how much area of the anode is imaged by each pixel of the CCD, and therefore the conversion from track length in pixels to $\mathrm{mm}$. This calibration is generally done with the CCDs at full readout resolution (unbinned) to provide the highest possible spatial resolution. The length calibration for the 4Shooter is $0.1604 \pm 0.0004 \mathrm{~mm} /$ pixel. In standard operations, the CCD is binned $4 \times 4$ 
and so each digitized channel of the CCD images a $0.642 \times 0.642 \mathrm{~mm}^{2}$ area of the anode.

The calibration proceeds by illuminating the detector interior with an LED and then identifying in the CCD images the pixel coordinates of the two machined channels that belong to the annular veto region. The LED is located in one of the camera mounts adjacent to the Canon SLR lens and points down toward the vacuum viewport. The LED can be turned on and off remotely, without disturbing the mechanical configuration of the detector.

This dataset is also used to determine the image transformation parameters required to form a single mosaic image of the amplification region from the four CCD images. Each image is translated and rotated. The rotation is chosen so the spacers in the amplification region are parallel to the $x$-axis of the mosaic image, and the translation is chosen such that fitted circles to the quadrants of the machined veto channels share a common center. Images from the LED-off data (where no TPC features are visible) are then stitched together using these transformations in order to form mosaic images as in Figure 6 .

\subsection{Gas gain}

An ${ }^{55} \mathrm{Fe}$ x-ray source is used to measure the gas gain as a function of anode bias voltage and gas pressure, and also to calibrate the energy scale of the charge readout electronics. The source $(30 \mu \mathrm{Ci})$ is placed on the cathode mesh, and the energy spectrum of the resulting photoelectric absorption events is recorded. For these low-energy events, a CR-112 charge-integrating amplifier with higher gain is used on the anode readout channel in place of the CR-113 amplifier. 

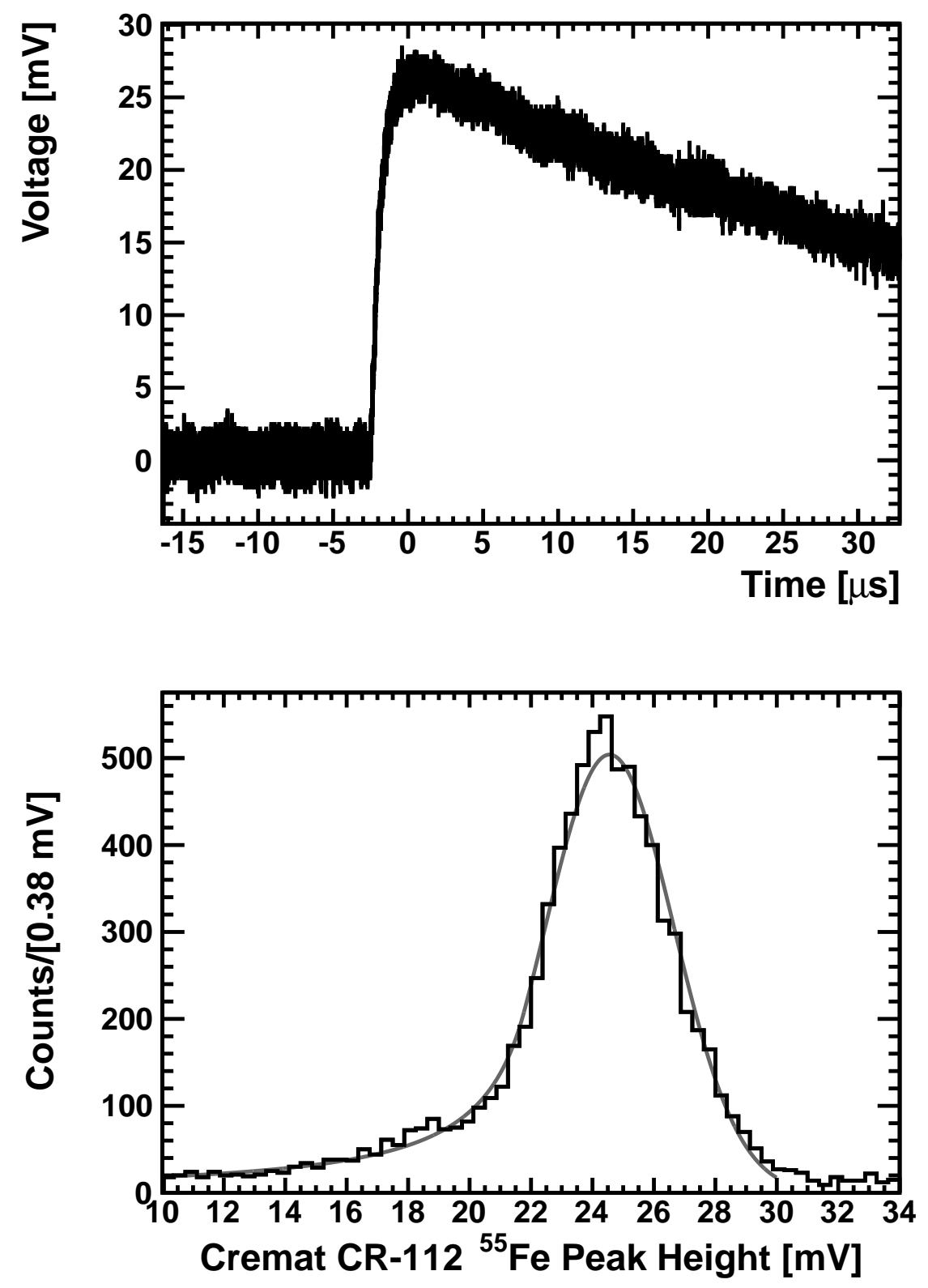

Figure 9: (Top): Sample waveform from an ${ }^{55} \mathrm{Fe}$ calibration exposure with 60 Torr $\mathrm{CF}_{4}$ and an anode voltage of $670 \mathrm{~V}$. (Bottom): Pulse height spectrum from the same calibration along with a fitted Crystal Ball function. For this spectrum $\sigma / \mu=8.5 \%$, where $\sigma$ is the standard deviation of the Gaussian portion of the fitted Crystal Ball function, and $\mu$ is the mean. 
A sample charge readout ${ }^{55} \mathrm{Fe}$ ionization event and the accumulated spectrum is shown in Figure 9. A Crystal Ball function [58] is fit to the spectrum, and the gas gain $G$ at that particular gas pressure and anode bias voltage is calculated from:

$$
G=\frac{W_{C F_{4}}}{\Delta E} \frac{V_{\text {peak }}}{e A}=2.75 \times 10^{3} V_{\text {peak }}[\mathrm{mV}],
$$

where $W_{C F_{4}}=34.3 \mathrm{eV}[59]$ is the $\mathrm{W}$-value of $\mathrm{CF}_{4}$ (but see comments below), $\Delta E$ is the ionization energy deposited in the detector (the energy of the ${ }^{55} \mathrm{Fe}$ x-ray), $V_{\text {peak }}$ is the voltage of the peak of the measured energy spectrum, $e=$ $1.60 \times 10^{-7} \mathrm{pC}$ is the elementary charge, and $A=13 \mathrm{mV} / \mathrm{pC}$ is the conversion gain of the CR-112 charge-integrating amplifier. The measured gas gain as a function of anode bias voltage for three different gas pressures $(45,60$ and 75 Torr) is shown in Figure 10. In all cases, the gas gain exceeds $10^{4}$, with a maximum gas gain of $10^{5}$ at 75 Torr. At a given anode voltage, the field in the amplification region is uniform, and the Townsend amplification factor is $\exp (\alpha d)$, where $\alpha$ is the Townsend coefficient, and $d$ is the amplification gap size (here $435 \mu \mathrm{m}$ ). In $\mathrm{CF}_{4}, \alpha$ grows linearly with electric field above $E / N=100 \times 10^{-17} \mathrm{~V} \mathrm{~cm}^{2}[52]$, and so the expected gas gain increases exponentially with anode voltage: $\exp \left(V_{\text {anode }} d\right)$. Figure 10 shows that the fits of the exponential function $\exp \left(a+b \cdot V_{\text {anode }}\right)$ match the data at each gas pressure (the constants $a$ and $b$ are free parameters for each data set).

We measure the stability of the gas gain as a function of time using a series of ${ }^{55} \mathrm{Fe}$ pulse height spectra over a 24 -hour period (see Figure 10), and find that the gas gain degrades by less than $3 \%$ over $24 \mathrm{~h}$, with an exponential decay time constant of $10.1 \mathrm{~h}$. Based on this measurement, we have chosen to evacuate and refill the 4Shooter with fresh $\mathrm{CF}_{4}$ gas once per day during 

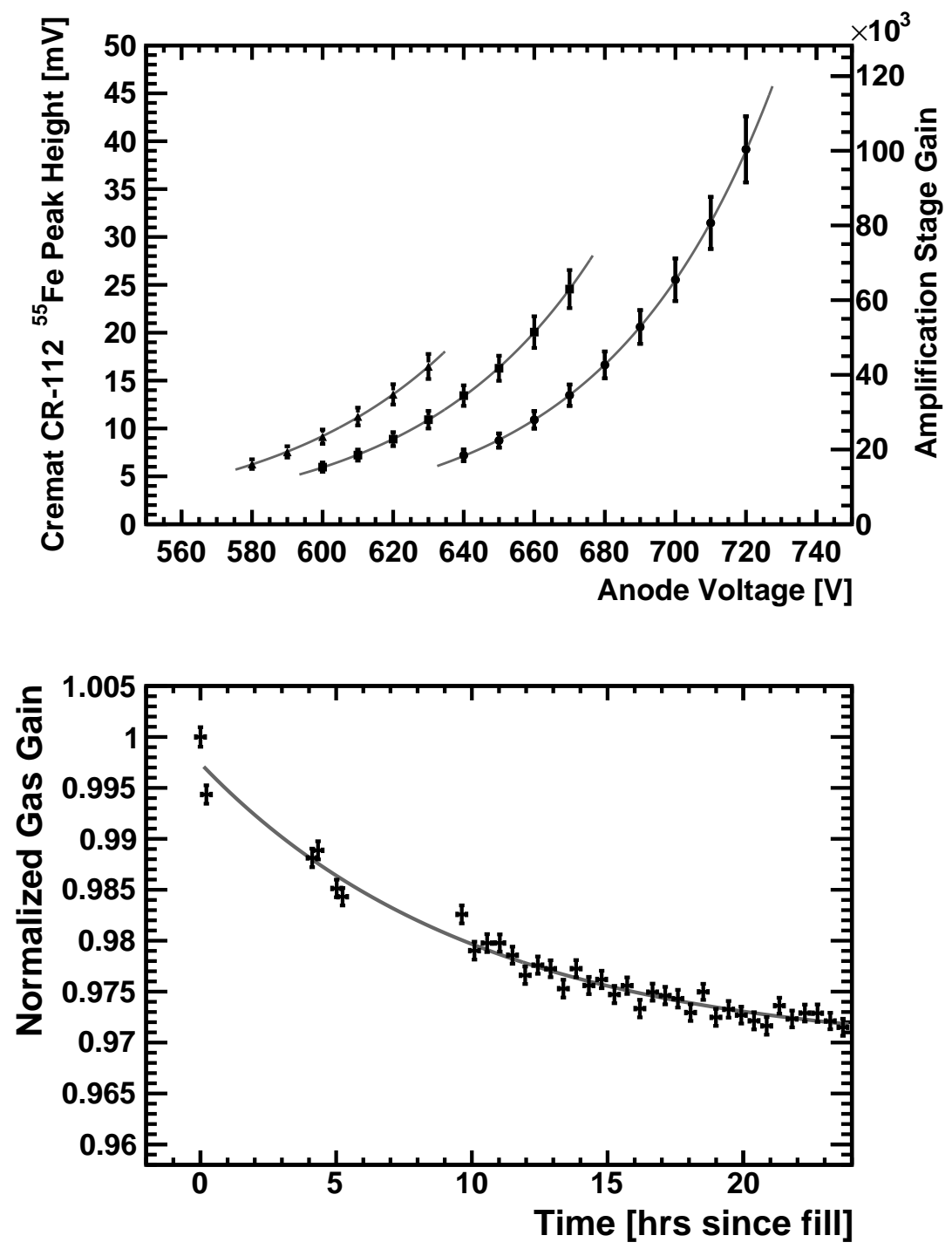

Figure 10: (Top) Gas gain versus voltage for three operating pressures (45, 60 and 75 Torr), along with fits of the exponential function $\exp \left(a+b \cdot V_{\text {anode }}\right)$. (Bottom) Gas gain as a function of time (normalized to the gain at $t=0$ ), along with an exponential plus constant offset fit: Gain $=a+\exp (b+c \cdot t[\mathrm{hr}])$ showing a gain degradation of $3 \%$ over one day. Data taken at a gas pressure of 75 Torr and an anode voltage of $720 \mathrm{~V}$. 
standard running operation. From ${ }^{55} \mathrm{Fe}$ data we also find that the gas gain is stable to $2 \%$ from gas fill to gas fill.

In this gas gain analysis, we have assumed a $W$-value for $\mathrm{CF}_{4}$ of $34.3 \mathrm{eV}$. There are discrepant values published in the literature, including $34.3 \mathrm{eV}$ [59] and $54 \mathrm{eV}[60,61]$. To help resolve this discrepancy, our group has carried out an independent measurement of $W_{C F_{4}}$ and found $W_{C F_{4}}=33.8 \pm 0.4 \mathrm{eV}$ [62].

We find that the measured gas gain decreases with x-ray flux, most likely due to the well-documented effect of space charge in the amplification gap [63]. At full source intensity, the measured gain was 30\% lower than at lower intensities. When making the gas gain measurements, we attenuated the source with layers of aluminum until the measured gas gain plateaued at a stable value. The data reported here used $150 \mu \mathrm{m}$ of aluminum. The ${ }^{55} \mathrm{Fe}$ produces $\mathrm{x}$-rays at three main energies $5.888,5.899$ and $6.49 \mathrm{keV}$ with relative intensities of $0.506,1.0$ and 0.176 , respectively [64]. Because the x-ray cross-sections in aluminum decrease with energy in this regime, we use the tabulated photon cross-sections in aluminum [65] to estimate the weighted average energy of the ${ }^{55} \mathrm{Fe}$ source after attenuation as $\Delta E=6.00 \mathrm{keV}_{\text {ee }}$. To explore the effect of source location on measured gas gain (and charge energy calibration), an additional study was done in which the $(x, y)$ location of the ${ }^{55} \mathrm{Fe}$ source was varied on the cathode mesh. The measured gas gain varied by less than $1 \%$ level with source location.

As the anode voltage is increased, the probability of spontaneous discharge in the amplification region (sparks) increases sharply, thereby limiting the achievable gas gain. At 60 Torr and $670 \mathrm{~V}$ on the anode (standard 
operating point), the discharge frequency is $5 \mathrm{mHz}$. These discharges produce intense scintillation light that can saturate pixels in the CCD camera and lead to spurious clusters of bright pixels in subsequent exposures [28]. Furthermore, each discharge initiates an interval of suppressed gas gain while the amplification region recharges with a measured recovery time constant of 3 seconds (set by the bandwidth of the anode high-voltage noise filter outside of the chamber). Events occurring during the recovery time are ignored. Further, at very high gas gains, tracks with large ionization density (e.g. nuclear recoils) can trigger sparks when Raether's limit is exceeded [66]. This sets a maximum stable operational gain for each combination of gas pressure and drift field.

\subsection{Charge energy calibration}

Using the energy calibration of the CR-112 amplifier, determined from the ${ }^{55} \mathrm{Fe}$ spectrum peak at $6 \mathrm{keV}$, the measured energies of x-ray quanta from ${ }^{241} \mathrm{Am}$ (specifically, the neptunium L-shell line emission at 13.9, 17.5 and $21.1 \mathrm{keV}$ [67]) agree with expectations at the $1 \%$ level. This calibration is transferred to the anode charge integrating amplifier (CR-113) by an independent measurement of the conversion gain ratio between the CR-113 and the CR-112 of $A_{113} / A_{112}$. The gain ratio was determined by matching the features of the source-free (background) spectra measured by each amplifier in the energy range $40-150 \mathrm{keV}$, under the same operating conditions (gas pressure and anode and cathode voltages). The background spectrum is a broken power law with a knee near $70 \mathrm{keV}$ (see Fig. 11). At an anode voltage of $670 \mathrm{~V}$ and a $\mathrm{CF}_{4}$ pressure of 60 Torr, a gain ratio of $A_{113} / A_{112}=0.112$ matches the features in the spectrum, and the total rates measured by the 


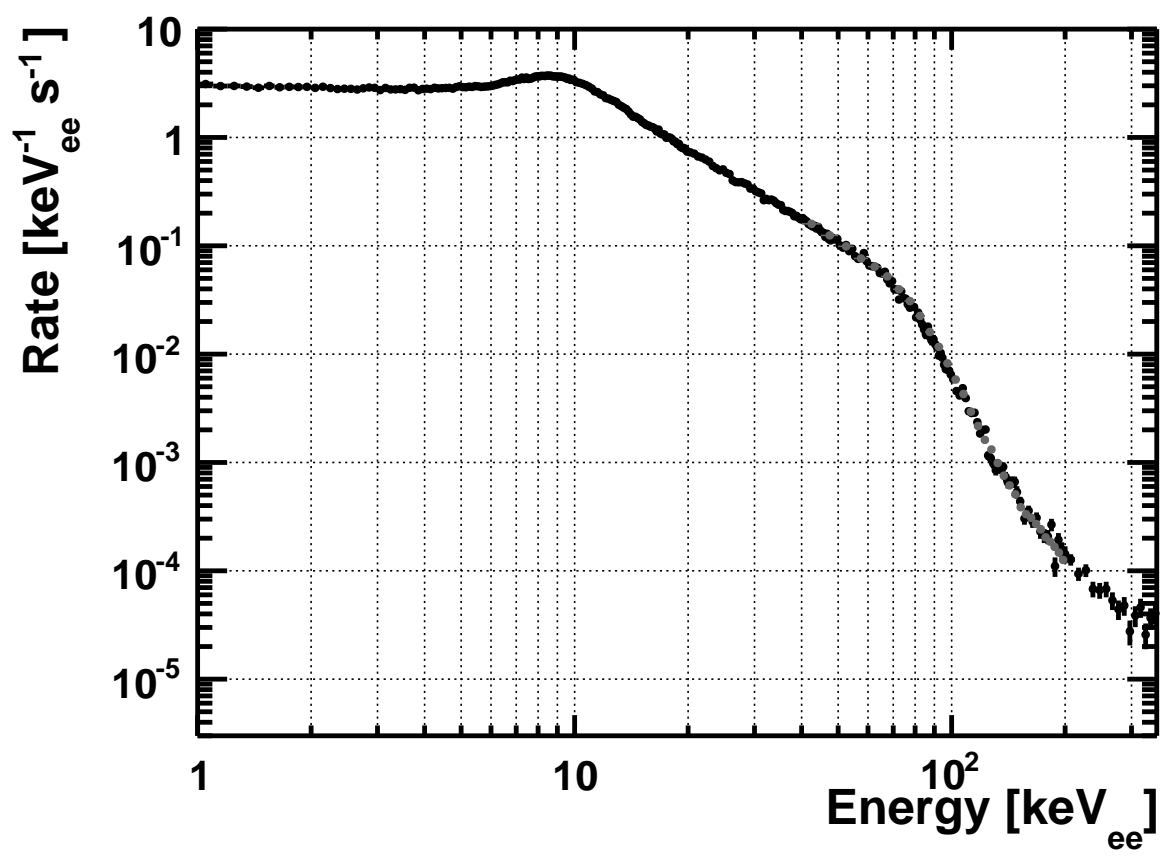

Figure 11: Background energy spectrum measured with the CR-112 charge integrating amplifier attached to the anode. The peak between 5 and $15 \mathrm{keV}_{\mathrm{ee}}$ is consistent with minimum-ionizing particles traversing the full vertical length of the detector. At energies higher than the peak, the spectrum is a power law $\left(E^{-2.15}\right)$, followed by a "knee" from 60 to $100 \mathrm{keV}_{\mathrm{ee}}$, and a steepened spectrum $\left(E^{-6.6}\right)$. The knee arises because electrons with energies higher than $\approx 60 \mathrm{keV}_{\text {ee }}$ are typically not fully contained in the detector. The ${ }^{55} \mathrm{Fe}$ energy calibration of the CR-112 preampifier is transferred to the CR-113 preamplifier by matching the observed background energy spectra in the vicinity of the knee. 
two amplifiers agree at the $1 \%$ level. Changing the gain ratio by $5 \%$ leads to significant differences between the measured spectra, and so we assign a $2.5 \%$ uncertainty to the gain ratio.

The conversion factor $g_{113}$ from $\mathrm{mV}$ to $\mathrm{keV}_{\mathrm{ee}}$ for the standard 4 Shooter configuration (CR-113 connected to the central anode) is then

$$
g_{113}\left[\mathrm{keV}_{\mathrm{ee}} / \mathrm{mV}\right]=\left(\frac{6.0 \mathrm{keV}_{\mathrm{ee}}}{V_{112}}\right)\left(\frac{A_{112}}{A_{113}}\right),
$$

where $V_{112}$ is the mean voltage of the ${ }^{55} \mathrm{Fe}$ spectrum measured with the CR112 amplifier (in $\mathrm{mV}$ ). At 60 Torr $\mathrm{CF}_{4}$ and an anode voltage of $670 \mathrm{~V}$, we find $g_{113}=2.2 \mathrm{keV}_{e e} / \mathrm{mV}$ with a total systematic uncertainty of approximately $4 \%$ and minimal statistical uncertainty [44].

\subsection{CCD noise measurements}

The two main noise sources intrinsic to CCDs are read noise and dark noise. The pixel values of a difference image of two same-duration dark exposures are distributed normally. The width $\sigma$ of that Gaussian distribution is equal to $\sqrt{2} \sigma_{N}$, where $\sigma_{N}$ is the total per-pixel noise in the CCD (nominally dominated by read and dark noise). When the 4Shooter CCDs are run in their native resolution (unbinned), we measure $\sigma_{N}$ in the range of 5 to $8 \mathrm{ADU}$ (the arbitrary digital units recorded by the camera - see Section 8.6 for the calibration from ADU to deposited energy in the detector), depending on the camera. Given the nominal CCD conversion gain of 1.3 to $1.65 e^{-/ A D U}$ (again, depending on the camera), this corresponds to 7 to $13 e^{-}$total noise.

To increase the signal to noise in a single image, we bin the CCDs $4 \times 4$ prior to digitization. We discovered, however, that $\sigma_{N}$ increases linearly with the CCD binning in the parallel direction, likely due to the so-called spurious 


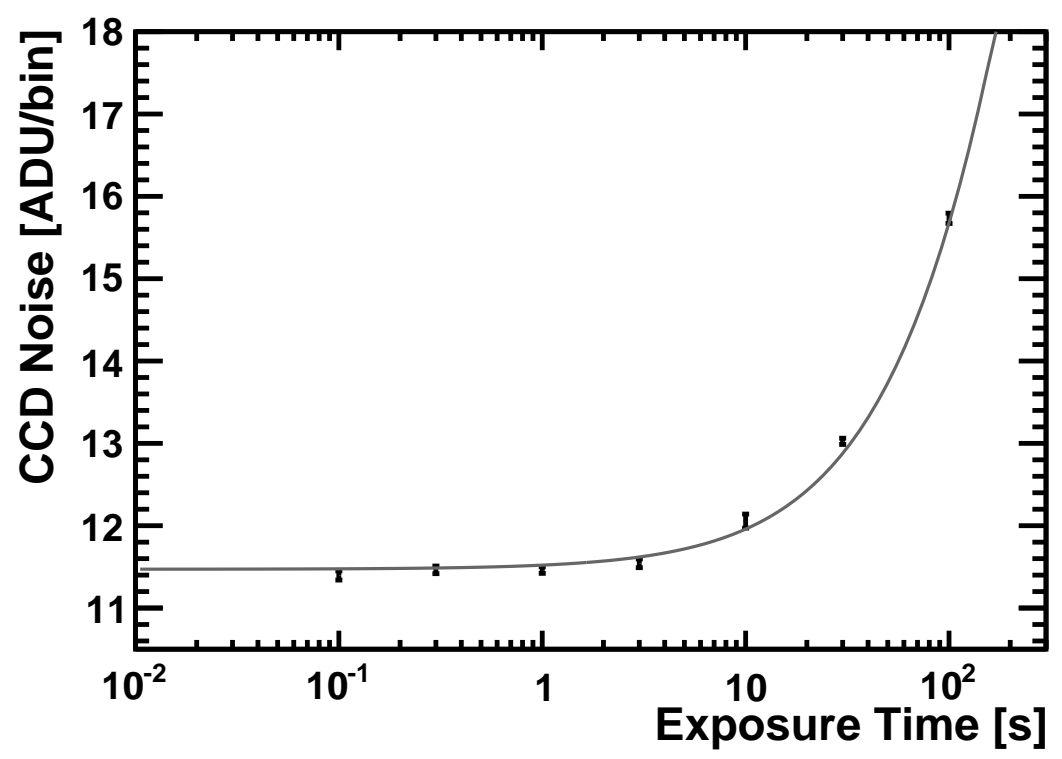

Figure 12: Total per-pixel noise for $4 \times 4$ binning in a single 4 Shooter CCD as a function of exposure time. Filled black circles are data, and the gray curve is a fit of $\sqrt{\sigma_{R}^{2}+R_{D} t}$ to the data. The dark noise contributes significantly to the total noise for exposure times above 10 seconds. These measurements were made by constructing difference images from pairs of dark exposures to remove fixed-pattern noise in the CCD. 
charge effect, as described in Ref. [68]. CCD vendors generally quote the noise for unbinned operation, and so we measure the noise vs. binning. At $4 \times 4$ binning, the CCD noise $\sigma_{N}$ ranges from 7 to $11 \mathrm{ADU}$, depending on the camera.

Figure 12 shows the dependence of $\sigma_{N}$ on exposure time $t$ for one CCD when binned $4 \times 4$. The leading contributions to the total noise are the read noise $\sigma_{R}$ and the dark noise $\sqrt{R_{D} t}$, where $R_{D}$ is the per-bin dark rate (ADU/sec), such that $\sigma_{N}=\sqrt{\sigma_{R}^{2}+R_{D} t}$. For short exposure times, the noise is read-noise dominated and therefore independent of exposure time. At the transition point $t \approx 10 \mathrm{~s}$, the dark noise is comparable to the read noise. A fit finds $\sigma_{R}=11.5 \pm 0.1 \mathrm{ADU}$ and $R_{D}=1.05 \pm 0.03 \mathrm{ADU} \mathrm{bin}{ }^{-1} \mathrm{sec}^{-1}$. These CCDs are operated at $-20^{\circ} \mathrm{C}$ using thermoelectric coolers but no cryogens. During standard operation, we restrict the CCD exposure times to be less than 10 seconds where $\sigma_{N} \approx \sigma_{R}$.

\subsection{Spatial variations in the CCD response}

The number of photons detected by the $\mathrm{CCD}$, per $\mathrm{keV}$ of ionization energy, varies spatially across the amplification region. There are many different causes for this non-uniformity, including variations in the amplification region gap and suppressed light production in the vicinity of the insulating spacers. Even if the amplification region provided uniform light production, the measured image would still show spatial variations in brightness due to the throughput of the optical system.

To take this effect into account during event reconstruction, we generate a gain map to measure the CCD energy calibration correction factor as a function of $(x, y)$ position. To obtain a gain map, one would ideally like to 
deposit a uniform distribution of ionization charge in the $(x, y)$ plane of the detector and image the resulting photons. As an approximation, we use a ${ }^{57} \mathrm{Co}$ source of $122 \mathrm{keV} \gamma$-rays, whose interaction length in the low-pressure $\mathrm{CF}_{4}$ gas is orders of magnitude greater than the dimensions of the TPC. For example, at 60 Torr, the interaction length is $240 \mathrm{~m}$. This produces an approximately uniform distribution of ionization across the amplification region. In any single CCD exposure, the detectable photon signal is very weak. However, by averaging together thousands of these exposures, we obtain an image showing the main features described above (Fig. 13). In practice, careful image and pixel selection criteria are applied to deal with sparks and background tracks in the detector, as well as hot pixels in the CCD. The resulting gain map is smoothed using a Gaussian bilateral filter [69] with a domain width of 12 pixels and a range width of 3 ADU. These filter parameter values were chosen heuristically, and validated through the resulting gain map's performance on data, as described quantitatively at the end of this section. The choice of the filter range width was driven by the desire to limit the leakage of anomalously high or low ADU-valued pixels into neighboring pixels in the filtered map, while the choice of the filter domain width was driven by the need to remove the pixel-to-pixel variations in the unfiltered map due to finite statistics. The filtered gain map was then normalized to the smoothed average pixel value for pixels at least 10 pixels within the boundary of the central anode, and further than 20 pixels away from a spacer.

The dominant spatial structure in the gain map arises from two main contributions: (1) suppressed system gain near the insulating spacers in the 


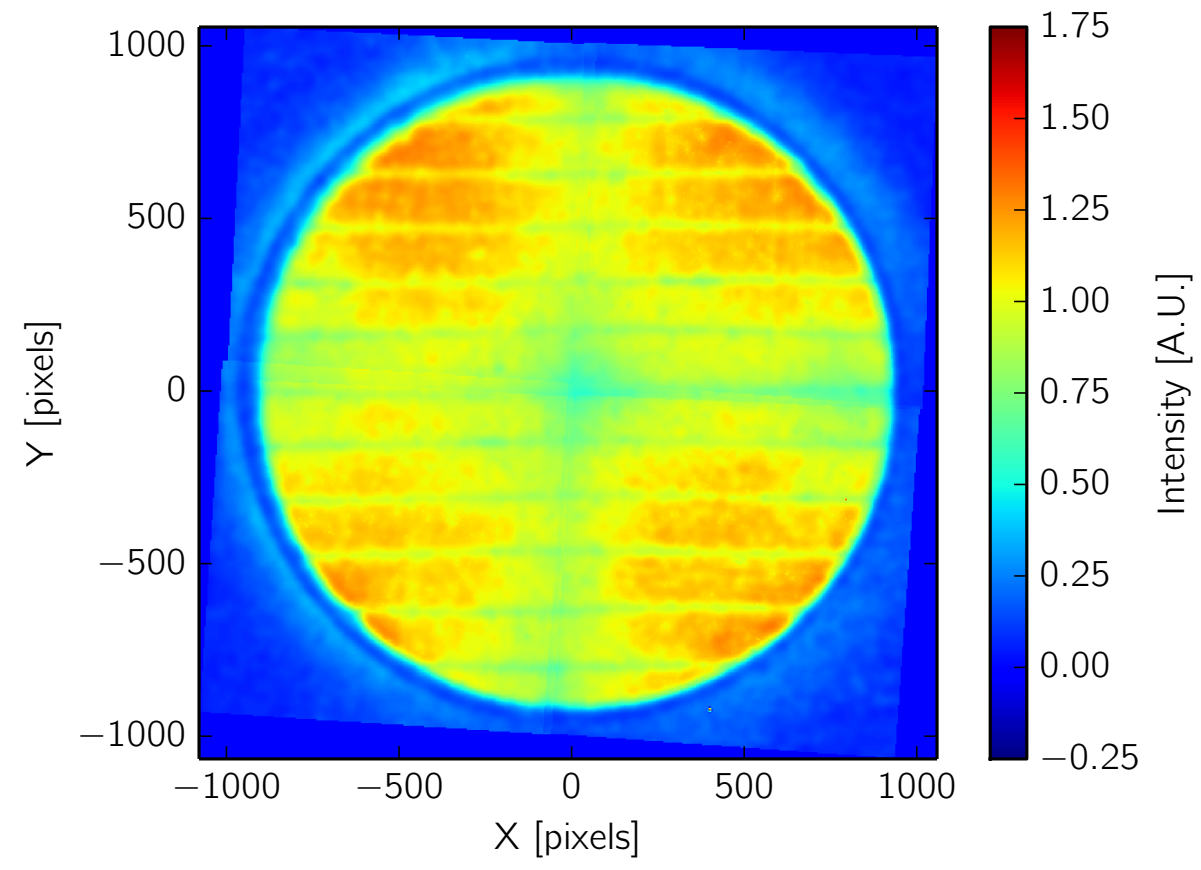

Figure 13: 4Shooter detector gain map from a long-duration ${ }^{57}$ Co source exposure used to calibrate the spatial variations in CCD gain. Larger values in the gain map correspond to regions of higher gain. The amplification stage spacers are clearly visible as locations of reduced gain, and for each camera, a radial fall-off in gain from vignetting is observed. The faint glow visible about the periphery of the circular amplification region arises from the reflections of photons off of the drift stage field shaping rings.

amplification region (see the 11 horizontal stripes in Fig. 13), and (2) vignetting in the optical system.

The suppressed response in the mosaic gain map image along the lines $x=$ 0 and $y=0$ arises from vignetting by the optical system. The characteristic signature of vignetting is a radial fall-off in the measured brightness in the field of view of each CCD camera. In Figure 13, these radial gradients are present in each individual CCD image in the mosaic. Detailed explanations 
of vignetting, along with fits of vignetting models to CCD data, are provided in Ref. [70].

The calibration of the CCD energy scale (see Section 8.6) relies on CCD measurements of long alpha tracks in the detector, and therefore uses the gain map described here. Before gain map correction, the energy of tracks determined from the CCD alone is more than 20\% less than the energy determined from charge alone on the periphery of images, where the effects of vignetting are most pronounced. After gain map correction, the CCDderived energy is consistent with the charge-derived energy independent of the radius at which tracks are reconstructed relative to the center of each CCD's image to within $3 \%$.

\subsection{CCD energy calibration}

The energy scale of the CCD cameras is determined by fitting the stopping versus range for collimated alpha tracks from an ${ }^{241} \mathrm{Am}$ source in Monte Carlo to data. For these alpha particles (which deposit $\approx 4.5 \mathrm{MeV}$ in the detector), SRIM simulations [71] show that more than $99 \%$ of the alpha energy goes into the ionization of the $\mathrm{CF}_{4}$ gas, and so this measurement determines the conversion factor from $\mathrm{ADU}$ to $\mathrm{keV}_{\mathrm{ee}}$. In order to further calibrate the energy scale for low-energy nuclear recoils, a measurement of the quenching factor is needed. The gas quenching quantifies what fraction of the recoil energy goes into ionization (as opposed to other forms of energy loss such as nuclear excitation). For a description of quenching in $\mathrm{CF}_{4}$ gas, see e.g. Refs. [61] and [72].

For the $\mathrm{ADU} / \mathrm{keV}_{\text {ee }}$ calibration, one collimated ${ }^{241} \mathrm{Am}$ source is placed in the field of view of each camera (see Figure 14). The source orientations 

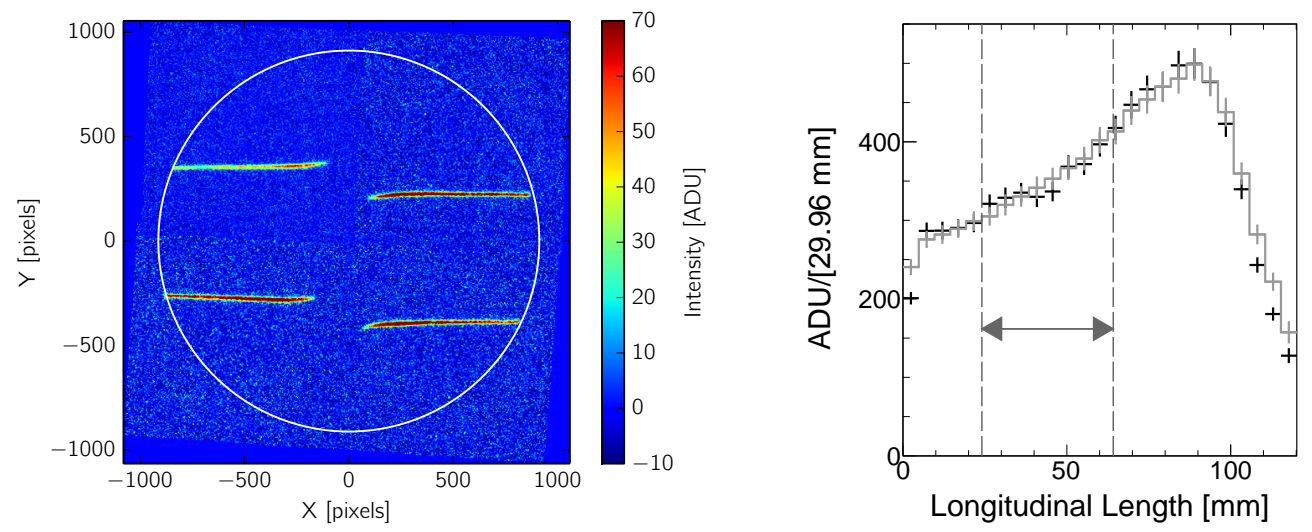

Figure 14: Left: four ${ }^{241} \mathrm{Am}$ alpha tracks emitted by sources in each of the four cameras during a typical alpha energy calibration. The exposures from each 4 Shooter camera have been stitched together to form a composite mosaic image, based on their known relative orientations. The dashed white circle shows the boundary of the central anode electrode of the amplification stage. Right: the average longitudinal projection of alpha tracks in a typical calibration dataset for one of the 4Shooter CCDs (black) compared with the tuned Monte Carlo prediction (gray), for data taken in 60 Torr $\mathrm{CF}_{4}$. The normalization of the Monte Carlo curve has been fit to the data to extract the total CCD gain in $\mathrm{ADU} / \mathrm{keV}_{\mathrm{ee}}$ within the bounds indicated. 
ensure that the emitted alpha particles travel horizontally (parallel to and above the amplification region), and are fully contained in a single camera's field of view. The collimators are further aligned such that the alpha tracks do not cross the fused silica spacers in the amplification region. A 1-second CCD exposure time was chosen such that the majority of images contained either zero or one alpha track in a single camera's field of view. A software cut on the reconstructed alpha track energies is used to remove images containing multiple, overlapping alpha tracks.

A series of data selection cuts are applied to the detected tracks to eliminate outliers in total range, energy, track angle, and straightness (relative to the collimator boresight). The reconstructed directions of alpha tracks in the data and Monte Carlo are required to be within $2^{\circ}$ of the nominal collimator boresight because multiple scattering is not presently modeled in our Monte Carlo. Reconstructed alpha tracks were discarded if their position and orientation was inconsistent with the known placement of the ${ }^{241} \mathrm{Am}$ sources. Additional data quality cuts were applied to ensure that no amplification region discharges (sparks) occurred in the 15 seconds prior to the relevant exposure. This 15 second waiting period was chosen to be $5 \times$ the measured time constant for gain recovery after discharge in ${ }^{55} \mathrm{Fe}$ x-ray measurements of the instantaneous gas gain. The input parameters to the Monte Carlo are then iteratively adjusted (see below) until the average longitudinal and transverse intensity profiles of the alpha tracks match the data.

The five input parameters that control the detector response are the transverse electron diffusion during drift, the $\mathrm{CCD}$ gain in $\mathrm{ADU} / \mathrm{keV}_{\mathrm{ee}}$ (this is the parameter under study), the gas quenching factor, the CCD length scale, and 
the CCD noise. The latter two parameters are constrained through independent measurements (see Sections 8.1 and 8.4), and are therefore fixed for all simulations. Although the effect of quenching is negligible for $5 \mathrm{MeV}$ alpha particles, for completeness, we model the amount of ionization deposited in the detector as [40]:

$$
\operatorname{LET}_{e l}\left(E_{r}\right)=S_{e}\left(E_{r}\right)+0.3 S_{n}\left(E_{r}\right),
$$

where $L E T_{e l}$ is the electronic energy deposited in the detector per unit length, and $E_{r}$ is the total energy of the alpha particle. $S_{e}$ and $S_{n}$ are the electronic and nuclear stopping, respectively, as predicted by SRIM-2006 [71]. The factor of 0.3 is chosen to be consistent with predictions by Hitachi [61] over a range of ion energies.

The total electron diffusion in the Monte Carlo is adjusted until the mean transverse width of the alpha tracks in data that pass all cuts is consistent with the mean transverse width of the simulated alpha tracks that pass all cuts. See Section 8.7 for further discussion of diffusion.

The mean and RMS energies of the alpha source in the Monte Carlo are initially fixed to the values measured for each source in vacuum with an ionimplanted silicon surface barrier detector. Only $\approx 4.5 \mathrm{MeV}$ of the initial alpha track energy remains after the alphas have straggled out of the thin foil covering the ${ }^{241} \mathrm{Am}$ in each source and through the approximately 1" long gas-filled collimator bore hole. The alpha source location in the Monte Carlo is defined relative to the active region of the TPC by the boresight line of the collimator and the starting position of alphas along that line. Only pixels within the active central anode region (see Section 5.2) are populated with Monte Carlo tracks. The collimator boresight line is defined from the 
orientation (angle and absolute position) of the data alpha tracks that pass all selection cuts. To account for the fact that the alpha sources are housed in collimators whose exit apertures do not lie exactly at the boundary of the active region of the detector, the initial energies of the alpha tracks in the Monte Carlo are adjusted until the mean longitudinal position of the alpha tracks' Bragg peaks agree with data. Adjusting the alpha track energies in the Monte Carlo implicitly assumes that a shift in the alpha track energies is equivalent to a shift in the alpha sources' positions, which is true in the limit that the alpha track energy variance is not dominated by straggling over the short inactive portion of the detector that the alphas must traverse between the ends of their collimators and the active region of the detector.

Once the source energy in the Monte Carlo has been tuned, the longitudinal projection of the tracks are computed in $\approx 5$-mm-wide bins (see right plot in Figure 14). To account for spatial variations in the gain, the data are normalized by the gain map (Section 8.5). The projections are averaged and compared between data and Monte Carlo. The system gain in $\mathrm{ADU} / \mathrm{keV}_{e e}$ in the Monte Carlo is iteratively tuned to achieve agreement with data in the region $\approx 24 \mathrm{~mm}$ after the start of the track and $\approx 24 \mathrm{~mm}$ before the Bragg peak. This interval was chosen for the fit such that the Bragg curves are approximately linear, in order to reduce systematic errors from improper alignment of the data and Monte Carlo longitudinal projections. According to SRIM, the alpha stopping in this region is approximately $30 \%$ lower than the ionization per unit length produced by a $100 \mathrm{keVr}$ fluorine nucleus. Table 1 lists the measured CCD energy calibration for each of the four CCD cameras for 60 Torr and $670 \mathrm{~V}$ anode bias. The resulting data and Monte 


\begin{tabular}{ll}
\hline \hline CCD \# & Gain $\left(\mathrm{ADU} / \mathrm{keV}_{\mathrm{ee}}\right)$ \\
\hline \hline 1 & $10.3 \pm 0.2$ \\
2 & $18.4 \pm 0.2$ \\
3 & $18.6 \pm 0.2$ \\
4 & $16.6 \pm 0.2$ \\
\hline
\end{tabular}

Table 1: The CCD energy scales in $\mathrm{ADU} / \mathrm{keV}_{\text {ee }}$ for the four 4 Shooter cameras measured with ${ }^{241} \mathrm{Am}$ tracks in 60 Torr $\mathrm{CF}_{4}$ with a $670 \mathrm{~V}$ anode bias. The gain measurement for each CCD has been averaged over data taken at a range of different heights $z$ and positions $(x, y)$ in the 4Shooter TPC. The error is the fit error on a constant fit to the data as a function of $z$. The RMS spread of the individual gain measurements at different positions and heights is observed to be less than $2 \%$.

Carlo comparison, after the gain is tuned, is shown in Figure 14 (right).

The differences between the camera gain values are partially due to differences in the intrinsic CCD conversion gains ( $e^{-} / \mathrm{ADU}$ ) between the cameras, and partially due to differences in optical throughput. Tests performed subsequent to the majority of the results presented in this paper determined that the conversion gain of the CCD with the lowest $\mathrm{ADU} / \mathrm{keV}_{\mathrm{ee}}$ gain, CCD \#1, was both anomalously large, and dependent on the intensity of illumination. This out of specification behavior largely accounts for its systematically lower $\mathrm{ADU} / \mathrm{keV}_{\mathrm{ee}}$ gain, relative to the other three cameras. Due to its abnormal performance, CCD \#1 has since been replaced.

By repeating this measurement with the alpha sources at a range of heights $z$ we verified that, as desired, the CCD gain calibration is insensitive to the electron drift distance $z$ at the $2 \%$ level. This sets an upper-limit on the electron loss during drift (from e.g. fringe fields in the TPC or attachment 
on to gas impurities), and also on the accuracy of the track energy reconstruction for diffuse tracks (long drift distance). The measured gain was also found to be spatially uniform in $(x, y)$ at the $1-2 \%$ level (after correcting for the gain map) from measurements performed with the alpha sources at eight distinct positions $(x, y)$ over the anode, although these studies did not sample the full field of view. This same set of data was used to measure the transverse electron diffusion, as described in the following section.

\subsection{Transverse electron diffusion}

The diffusion of the drifting electrons in the TPC sets a limit on the total viable length $L$ of the drift region of the TPC before the transverse diffusion exceeds the track length, and therefore compromises the directional sensitivity of the instrument. For example, fluorine recoils of energy $40 \mathrm{keV}_{r}$ travel $1 \mathrm{~mm}$ in 60 Torr $\mathrm{CF}_{4}$, which sets the scale for allowable diffusion. Previous measurements of electron diffusion in $\mathrm{CF}_{4}$ gas $[52,73]$ show that at 60 Torr and the drift field that minimizes transverse diffusion $(225 \mathrm{~V} / \mathrm{cm})$ the RMS track width reaches $1 \mathrm{~mm}$ after $25 \mathrm{~cm}$ of drift. We define the RMS track width as the square root of the transverse moment of the track, and the transverse moment as the second central moment of the track. To calculate the second central moment of the track, we first determine the track axis, and then calculate the intensity-weighted sum of the squares of the distances of each pixel in the track from the track axis.

In this work, measurements of $D_{T} / \mu$, the ratio of the electron transverse diffusion constant to the electron mobility in $\mathrm{CF}_{4}$ gas, have been made insitu, as a function of the ratio of the drift electric field to the number density of gas molecules $E / N$. These measurements were carried out using the same 
collimated alpha sources and track selection criteria from the energy calibration measurements described in Section 8.6. This time the sources were inserted into the detector at a range of heights $z$. As described in [73], the measured transverse moment $\sigma_{T}^{2}$ of these alpha tracks grows linearly with $z$ because of electron diffusion:

$$
\sigma_{T}^{2}(z)=\sigma_{T, 0}^{2}+2\left(\frac{D_{T}}{\mu}\right)\left(\frac{z L}{V}\right),
$$

where $\sigma_{T, 0}^{2}$ is the transverse moment for zero drift length, and $V$ is the applied drift field voltage (the cathode voltage).

To simulate the effect of transverse diffusion from drift, the primary ionization tracks in the Monte Carlo are spatially convolved with a Gaussian kernel of width $\sigma_{T}^{M C}$ prior to the simulated CCD digitization. This spatial smearing accounts primarily for diffusion, but also for the imperfect focus of the CCD cameras and the intrinsic widths of the track-induced avalanches in the amplification stage of the detector. Measurements of $D_{T} / \mu$ are obtained by adjusting the gaussian width $\sigma_{T}^{M C}$ applied to collimated alpha tracks in Monte Carlo until the mean transverse moment $\sigma_{T}^{2}$ of the reconstructed Monte Carlo alpha tracks matches the mean transverse moment of the reconstructed alpha tracks in data for a range of drift distances $z$ (at constant gas pressure and anode and drift bias voltages).

Data was taken at a gas pressure of 60 Torr and an anode bias voltage of $635 \mathrm{~V}$. The transverse moment used in Monte Carlo $\left(\sigma_{T}^{M C}\right)^{2}$ required to obtain agreement for $\sigma_{T}^{2}$ between data and Monte Carlo is shown in Figure 15 versus drift height $z$, along with the fit of Equation 5. Unlike in Ref. [73], fitting Equation 5 to $\left(\sigma_{T}^{M C}\right)^{2}$ instead of to the observed transverse moment $\left(\sigma_{T}\right)^{2}$ decouples the diffusion measurement from possible bias introduced by 
the digitization and readout. The values of $D_{T} / \mu$ (see Table 2) can be calculated from the slope of the fitted lines, and agree with the published value of $D_{T} / \mu=0.051 \mathrm{~V}$ for $E / N=9.5 \mathrm{Td}$ [52]. The errors have been computed as the difference in values obtained for $D_{T} / \mu$ and $\sigma_{T, 0}^{M C}$ between the results of this analysis applied to Monte Carlo data and the known input values. The $y$-intercept of the fitted lines represents the inferred intrinsic, heightindependent transverse width of the alpha tracks, prior to CCD digitization and readout. The zero-drift-length transverse widths listed in Table 2 are comparable across each of the four CCDs, with variations arising because these parameters depend not only on the width of the alpha tracks in absence of diffusion, but also on a number of effects presently not incorporated in the Monte Carlo simulation including the secondary electron avalanche width, the amplification stage grid spacing $(254 \mu \mathrm{m})$, the imperfect focus of each CCD, and lateral straggling of the alphas themselves, with the latter expected to be dominant. None of these effects, however, are expected to vary with source height. This assumption is validated by the similar slopes of transverse moment vs. alpha source height for all four CCD cameras (see Figure 15).

\section{Conclusions}

The 4Shooter detector has been built and commissioned in a surface laboratory at MIT. The detector performance, including the CCD and charge readout energy calibrations, gas gain measurements and transverse electron diffusion, has been described. Additional studies are underway, including a neutron calibration run to measure the track angle reconstruction resolution 


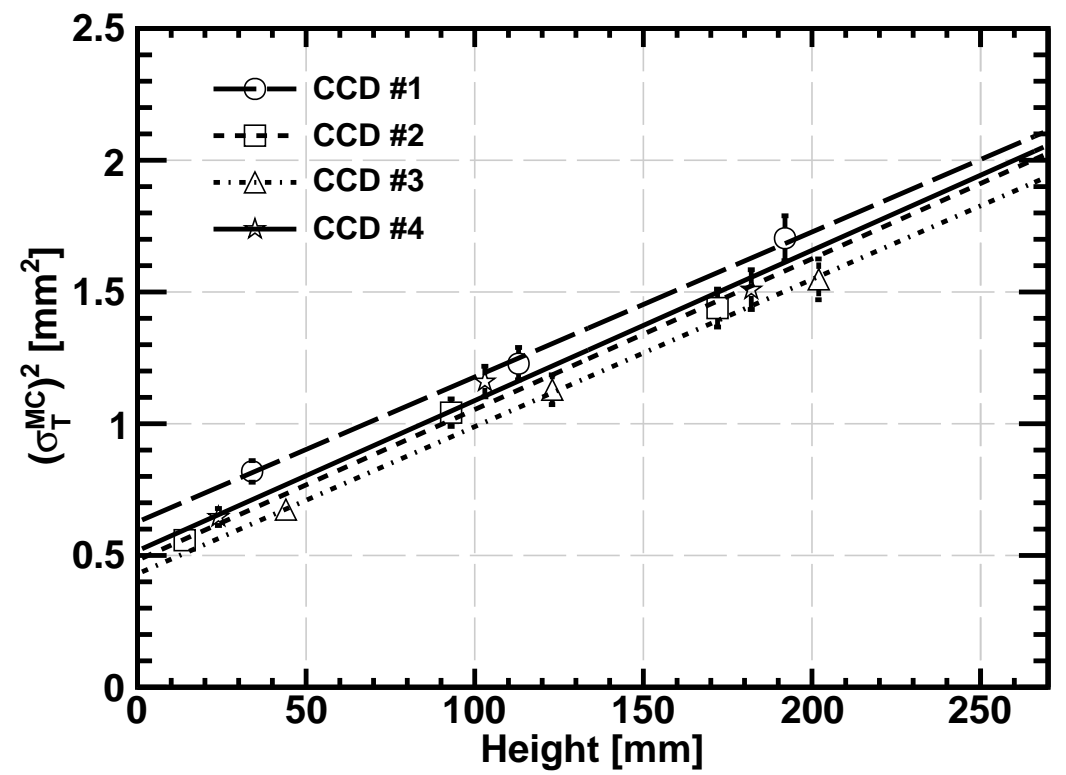

Figure 15: The spatial gaussian smearing before digitization and readout required in the Monte Carlo to match the mean reconstructed transverse moment of alpha tracks in the data. For this study, alpha sources were placed at varying heights $z$ above the amplification stage in the 4Shooter in 60 Torr $\mathrm{CF}_{4}$ with a $635 \mathrm{~V}$ anode bias and a $187 \mathrm{~V} / \mathrm{cm}$ drift field. The lines represent fits of Equation 5 to the data for each CCD separately. 


\begin{tabular}{lll}
\hline \hline $\mathrm{CCD} \#$ & $D_{T} / \mu[\mathrm{V}]$ & $\sigma_{T, 0}^{M C}[\mathrm{~mm}]$ \\
\hline \hline 1 & $0.052 \pm 0.005$ & $0.79 \pm 0.05$ \\
2 & $0.054 \pm 0.005$ & $0.69 \pm 0.04$ \\
3 & $0.052 \pm 0.005$ & $0.66 \pm 0.07$ \\
4 & $0.053 \pm 0.005$ & $0.72 \pm 0.05$ \\
\hline \hline
\end{tabular}

Table 2: The electron transverse diffusion constant $D_{T} / \mu$ and the inferred transverse width of the alpha tracks at zero drift length prior to CCD readout and digitization $\sigma_{T, 0}^{M C}$, based on a fit to Equation 5, as described in the text. Data was taken in 60 Torr $\mathrm{CF}_{4}$ with a $635 \mathrm{~V}$ anode bias and a $187 \mathrm{~V} / \mathrm{cm}$ drift field. The errors have been estimated from comparing the results of the calibration procedure on purely Monte Carlo datasets to the known input diffusion. This result agrees with the published value of $D_{T} / \mu=0.051 \mathrm{~V}$ for our operating point of $E / N=9.5 \mathrm{Td}[52]$.

and head-tail reconstruction efficiency at low recoil energies.

The 4Shooter detector was designed as a prototype for the cubic-meter scale detector (DMTPCino), not to set competitive limits on WIMP-proton spin-dependent interactions. That said, we have shown [43] that the 4Shooter nuclear recoil detection efficiency is $50 \%$ at $50 \mathrm{keV}_{r}$, which gives a spindependent WIMP-proton cross-section reach of $5 \times 10^{-37} \mathrm{~cm}^{2}$ at a WIMP mass of $100 \mathrm{GeV} / \mathrm{c}^{2}$ if run background-free for one live-year. Under the same conditions, the DMTPCino detector sensitivity would be a factor of 50 better $\left(1 \times 10^{-38} \mathrm{~cm}^{2}\right)$, and comparable to the current leading limits from COUPP [74] and SIMPLE [75]. 


\section{Acknowledgements}

PF acknowledges support from the US Department of Energy (contract number DE-FG02-05ER41360). SV and IJ acknowledge support from the U.S. Department of Homeland Security under Award Number 2011-DN-077ARI050-03 and the U.S. Department of Energy under Award Number DESC0007852. JM, RE, and GS acknowledge support from the Science and Technology Facilities Council under Award Number ST/K502261/1, and Royal Holloway University of London. We also acknowledge the support of the the Pappalardo Fellowship program at MIT, the MIT Kavli Institute for Astrophysics and Space Research, and the Physics Department at the Massachusetts Institute of Technology. We would like to thank Ulrich Becker (MIT) for useful discussions and generosity with hardware. We also acknowledge the valuable technical assistance of Mike Grossman and Mark Belanger (MIT), and Ernie Ihloff, Jim Kelsey, Chris Vidal and Peter Binns at the MIT Bates Research and Engineering Center for support with the vacuum system. Additionally, we acknowledge electrical engineering support from Jim MacArthur at the Harvard Instrument Design Laboratory. Finally, we thank the anonymous reviewer for detailed comments on this work.

[1] Planck Collaboration, P. A. R. Ade, N. Aghanim, C. Armitage-Caplan, M. Arnaud, M. Ashdown, F. Atrio-Barandela, J. Aumont, C. Baccigalupi, A. J. Banday, et al., Planck 2013 results. XVI. Cosmological parameters, ArXiv e-prints (2013).

[2] G. Bertone, D. Hooper, J. Silk, Particle dark matter: Evidence, candidates and constraints, Phys. Rep. 405 (2005) 279-390. 
[3] J. L. Feng, Dark Matter Candidates from Particle Physics and Methods of Detection, Ann. Rev. Astron. Astrophys. 48 (2010) 495-545.

[4] M. W. Goodman, E. Witten, Detectability of certain dark-matter candidates, Phys. Rev. D31 (1985) 3059.

[5] R. J. Gaitskell, Direct Detection of Dark Matter, Ann. Rev. Nucl. Phys. 54 (2004) 315-359.

[6] K. Freese, J. A. Frieman, A. Gould, Signal Modulation in Cold Dark Matter Detection, Phys. Rev. D37 (1988) 3388.

[7] K. Freese, M. Lisanti, C. Savage, Colloquium: Annual modulation of dark matter, Rev. Mod. Phys. 85 (2013) 1561-1581.

[8] R. Bernabei, P. Belli, F. Cappella, V. Caracciolo, S. Castellano, R. Cerulli, C. J. Dai, A. d'Angelo, S. d'Angelo, A. Di Marco, H. L. He, A. Incicchitti, H. H. Kuang, X. H. Ma, F. Montecchia, D. Prosperi, X. D. Sheng, R. G. Wang, Z. P. Ye, Final model independent result of DAMA/LIBRA-phase1, European Physical Journal C 73 (2013) 2648.

[9] C. Aalseth, P. Barbeau, J. Colaresi, J. Collar, J. Diaz Leon, et al., Search for an Annual Modulation in a P-type Point Contact Germanium Dark Matter Detector, Phys. Rev. Lett. 107 (2011) 141301.

[10] CDMS Collaboration, R. Agnese, et al., Dark Matter Search Results Using the Silicon Detectors of CDMS II, ArXiv e-prints (2013).

[11] LUX Collaboration, D. S. Akerib, H. M. Araujo, X. Bai, A. J. Bailey, J. Balajthy, S. Bedikian, E. Bernard, A. Bernstein, A. Bolozdynya, 
A. Bradley, D. Byram, S. B. Cahn, M. C. Carmona-Benitez, C. Chan, J. J. Chapman, A. A. Chiller, C. Chiller, K. Clark, T. Coffey, A. Currie, A. Curioni, S. Dazeley, L. de Viveiros, A. Dobi, J. Dobson, E. M. Dragowsky, E. Druszkiewicz, B. Edwards, C. H. Faham, S. Fiorucci, C. Flores, R. J. Gaitskell, V. M. Gehman, C. Ghag, K. R. Gibson, M. G. D. Gilchriese, C. Hall, M. Hanhardt, S. A. Hertel, M. Horn, D. Q. Huang, M. Ihm, R. G. Jacobsen, L. Kastens, K. Kazkaz, R. Knoche, S. Kyre, R. Lander, N. A. Larsen, C. Lee, D. S. Leonard, K. T. Lesko, A. Lindote, M. I. Lopes, A. Lyashenko, D. C. Malling, R. Mannino, D. N. McKinsey, D.-M. Mei, J. Mock, M. Moongweluwan, J. Morad, M. Morii, A. S. J. Murphy, C. Nehrkorn, H. Nelson, F. Neves, J. A. Nikkel, R. A. Ott, M. Pangilinan, P. D. Parker, E. K. Pease, K. Pech, P. Phelps, L. Reichhart, T. Shutt, C. Silva, W. Skulski, C. J. Sofka, V. N. Solovov, P. Sorensen, T. Stiegler, K. O‘Sullivan, T. J. Sumner, R. Svoboda, M. Sweany, M. Szydagis, D. Taylor, B. Tennyson, D. R. Tiedt, M. Tripathi, S. Uvarov, J. R. Verbus, N. Walsh, R. Webb, J. T. White, D. White, M. S. Witherell, M. Wlasenko, F. L. H. Wolfs, M. Woods, C. Zhang, First results from the LUX dark matter experiment at the Sanford Underground Research Facility, ArXiv e-prints (2013).

[12] E. Aprile, M. Alfonsi, K. Arisaka, F. Arneodo, C. Balan, L. Baudis, B. Bauermeister, A. Behrens, P. Beltrame, K. Bokeloh, E. Brown, G. Bruno, R. Budnik, J. M. R. Cardoso, W.-T. Chen, B. Choi, D. Cline, A. P. Colijn, H. Contreras, J. P. Cussonneau, M. P. Decowski, E. Duchovni, S. Fattori, A. D. Ferella, W. Fulgione, F. Gao, M. Garbini, C. Ghag, K.-L. Giboni, L. W. Goetzke, C. Grignon, E. Gross, W. Ham- 
pel, F. Kaether, A. Kish, J. Lamblin, H. Landsman, R. F. Lang, M. Le Calloch, C. Levy, K. E. Lim, Q. Lin, S. Lindemann, M. Lindner, J. A. M. Lopes, K. Lung, T. Marrodán Undagoitia, F. V. Massoli, A. J. Melgarejo Fernandez, Y. Meng, A. Molinario, E. Nativ, K. Ni, U. Oberlack, S. E. A. Orrigo, E. Pantic, R. Persiani, G. Plante, N. Priel, A. Rizzo, S. Rosendahl, J. M. F. dos Santos, G. Sartorelli, J. Schreiner, M. Schumann, L. Scotto Lavina, P. R. Scovell, M. Selvi, P. Shagin, H. Simgen, A. Teymourian, D. Thers, O. Vitells, H. Wang, M. Weber, C. Weinheimer, Dark Matter Results from 225 Live Days of XENON100 Data, Physical Review Letters 109 (2012) 181301.

[13] E. Armengaud, C. Augier, A. Benoît, L. Bergé, T. Bergmann, J. Blümer, A. Broniatowski, V. Brudanin, B. Censier, M. Chapellier, F. Charlieux, F. Couëdo, P. Coulter, G. A. Cox, J. Domange, A. A. Drillien, L. Dumoulin, K. Eitel, D. Filosofov, N. Fourches, J. Gascon, G. Gerbier, J. Gironnet, M. Gros, S. Henry, G. Heuermann, S. Hervé, A. Juillard, M. Kleifges, H. Kluck, V. Kozlov, H. Kraus, V. A. Kudryavtsev, H. Le Sueur, P. Loaiza, S. Marnieros, A. Menshikov, X.-F. Navick, C. Nones, E. Olivieri, P. Pari, B. Paul, M. Robinson, S. Rozov, V. Sanglard, B. Schmidt, B. Siebenborn, D. Tcherniakhovski, A. S. Torrento-Coello, L. Vagneron, R. J. Walker, M. Weber, E. Yakushev, X. Zhang, Search for low-mass WIMPs with EDELWEISS-II heat-and-ionization detectors, Phys. Rev. D 86 (2012) 051701.

[14] Z. Ahmed, D. S. Akerib, S. Arrenberg, C. N. Bailey, D. Balakishiyeva, L. Baudis, D. A. Bauer, P. L. Brink, T. Bruch, R. Bunker, B. Cabrera, 
D. O. Caldwell, J. Cooley, E. Do Couto E Silva, P. Cushman, M. Daal, F. Dejongh, P. di Stefano, M. R. Dragowsky, L. Duong, S. Fallows, E. Figueroa-Feliciano, J. Filippini, J. Fox, M. Fritts, S. R. Golwala, J. Hall, R. Hennings-Yeomans, S. A. Hertel, D. Holmgren, L. Hsu, M. E. Huber, O. Kamaev, M. Kiveni, M. Kos, S. W. Leman, S. Liu, R. Mahapatra, V. Mandic, K. A. McCarthy, N. Mirabolfathi, D. Moore, H. Nelson, R. W. Ogburn, A. Phipps, M. Pyle, X. Qiu, E. Ramberg, W. Rau, A. Reisetter, R. Resch, T. Saab, B. Sadoulet, J. Sander, R. W. Schnee, D. N. Seitz, B. Serfass, K. M. Sundqvist, M. Tarka, P. Wikus, S. Yellin, J. Yoo, B. A. Young, J. Zhang, Results from a Low-Energy Analysis of the CDMS II Germanium Data, Phys. Rev. Lett. 106 (2011) 131302.

[15] D. N. Spergel, The motion of the Earth and the detection of WIMPs, Phys. Rev. D37 (1988) 1353.

[16] A. M. Green, B. Morgan, Optimizing WIMP directional detectors, Astropart. Phys. 27 (2007) 142-149.

[17] B. Morgan, A. M. Green, N. J. C. Spooner, Directional statistics for WIMP direct detection, Phys. Rev. D71 (2005) 103507.

[18] B. Morgan, A. M. Green, Directional statistics for WIMP direct detection. 2. 2D read-out, Phys. Rev. D72 (2005) 123501.

[19] J. Billard, F. Mayet, D. Santos, Exclusion limits from data of directional Dark Matter detectors, Phys. Rev. D82 (2010) 055011.

[20] J. Billard, F. Mayet, J. Macias-Perez, D. Santos, Directional detection 
as a strategy to discover galactic Dark Matter, Phys. Lett. B691 (2010) 156-162.

[21] J. Billard, F. Mayet, D. Santos, Markov chain Monte Carlo analysis to constrain dark matter properties with directional detection, Phys. Rev. D 83 (2011) 075002.

[22] S. Ahlen, N. Afshordi, J. Battat, J. Billard, N. Bozorgnia, et al., The case for a directional dark matter detector and the status of current experimental efforts, Int. J. Mod. Phys. A25 (2010) 1-51.

[23] K. N. Buckland, M. J. Lehner, G. E. Masek, M. Mojaver, Low pressure gaseous detector for particle dark matter, Phys. Rev. Lett. 73 (1994) $1067-1070$.

[24] P. Belli, R. Bernabei, C. Bacci, A. Incicchitti, D. Prosperi, Identifying a "dark matter" signal by nonisotropic scintillation detector, Nuovo Cimento C Geophysics Space Physics C 15 (1992) 473-479.

[25] N. J. C. Spooner, J. W. Roberts, D. R. Tovey, Measurements of Carbon Recoil Scintillation Efficiency and Anisotropy in Stilbene for WIMP Searches with Direction Sensitivity, in: J. J. C. Spooner (Ed.), Identification of Dark Matter, 1997, p. 481.

[26] Y. Shimizu, M. Minowa, H. Sekiya, Y. Inoue, Directional scintillation detector for the detection of the wind of WIMPs, Nuclear Instruments and Methods in Physics Research A 496 (2003) 347-352.

[27] T. Naka, T. Asada, T. Katsuragawa, K. Hakamata, M. Yoshimoto, K. Kuwabara, M. Nakamura, O. Sato, T. Nakano, Y. Tawara, G. De 
Lellis, C. Sirignano, N. D'Ambrossio, Fine grained nuclear emulsion for higher resolution tracking detector, Nuclear Instruments and Methods in Physics Research A 718 (2013) 519-521.

[28] S. Ahlen, J. Battat, T. Caldwell, C. Deaconu, D. Dujmic, et al., First Dark Matter Search Results from a Surface Run of the 10-L DMTPC Directional Dark Matter Detector, Phys.Lett. B695 (2011) 124-129.

[29] S. Vahsen, H. Feng, M. Garcia-Sciveres, I. Jaegle, J. Kadyk, et al., The Directional Dark Matter Detector $\left(D^{3}\right)$, EAS Publ.Ser. 53 (2012) 43-50.

[30] E. Daw, A. Dorofeev, J. Fox, J. Gauvreau, C. Ghag, et al., The DRIFT Directional Dark Matter Experiments, EAS Publ.Ser. 53 (2012) 11-18.

[31] D. Santos, J. Billard, G. Bosson, J. L. Bouly, O. Bourrion, C. Fourel, O. Guillaudin, J. Lamblin, J. F. Muraz, F. Mayet, J. P. Richer, Q. Riffard, E. Ferrer, I. Giomataris, F. J. Iguaz, L. Lebreton, D. Maire, MIMAC: A micro-tpc matrix for dark matter directional detection, ArXiv e-prints (2013).

[32] K. Nakamura, K. Miuchi, S. Iwaki, H. Kubo, T. Mizumoto, H. Nishimura, J. D. Parker, T. Sawano, A. Takada, T. Tanimori, H. Sekiya, A. Takeda, NEWAGE, Journal of Physics Conference Series 375 (2012) 012013.

[33] E. Daw, J. R. Fox, J.-L. Gauvreau, C. Ghag, L. J. Harmon, M. Gold, E. R. Lee, D. Loomba, E. H. Miller, A. S. Murphy, S. M. Paling, J. M. Landers, M. Pipe, K. Pushkin, M. Robinson, D. P. Snowden-Ifft, N. J. C. 
Spooner, D. Walker, Spin-dependent limits from the DRIFT-IId directional dark matter detector, Astroparticle Physics 35 (2012) 397-401.

[34] K. Miuchi, H. Nishimura, K. Hattori, N. Higashi, C. Ida, S. Iwaki, S. Kabuki, H. Kubo, S. Kurosawa, K. Nakamura, J. Parker, T. Sawano, M. Takahashi, T. Tanimori, K. Taniue, K. Ueno, H. Sekiya, A. Takeda, K. Tsuchiya, A. Takada, First underground results with NEWAGE-0.3a direction-sensitive dark matter detector, Phys. Lett. B 686 (2010) 11-17.

[35] D. R. Nygren, Columnar recombination: a tool for nuclear recoil directional sensitivity in a xenon-based direct detection WIMP search, in: Journal of Physics Conference Series, volume 460 of Journal of Physics Conference Series, 2013.

[36] A. Drukier, K. Freese, D. Spergel, C. Cantor, G. Church, T. Sano, New Dark Matter Detectors using DNA for Nanometer Tracking, ArXiv eprints (2012).

[37] D. N. McKinsey, W. H. Lippincott, J. A. Nikkel, W. G. Rellergert, Trace detection of metastable helium molecules in superfluid helium by laser-induced fluorescence, Phys. Rev. Lett. 95 (2005) 111101.

[38] F. Cappella, R. Bernabei, P. Belli, V. Caracciolo, R. Cerulli, F. A. Danevich, A. d'Angelo, A. Di Marco, A. Incicchitti, D. V. Poda, V. I. Tretyak, On the potentiality of the $\mathrm{ZnWO}_{4}$ anisotropic detectors to measure the directionality of Dark Matter, European Physical Journal C 73 (2013) 2276. 
[39] D. Dujmic, et al. (DMTPC), Observation of the "head-tail" effect in nuclear recoils of low-energy neutrons, Nucl. Ins. Meth. Phys. Res. A592 (2008) 123.

[40] D. Dujmic, et al. (DMTPC), Charge amplification concepts for direction-sensitive dark matter detectors, Astropart. Phys. 30 (2008) $58-64$.

[41] G. Sciolla, DMTPC Collaboration, The DMTPC project, J. Phys. Conf. Ser. 179 (2009) 012009.

[42] J. Lopez, D. Dujmic, S. Ahlen, J. B. C. Deaconu, P. Fisher, et al., Background Rejection in the DMTPC Dark Matter Search Using Charge Signals, Nucl. Instrum. Meth. A696 (2012) 121-128.

[43] S. Henderson, An assessment of the sensitivity of a low-pressure Time Projection Chamber to the direction of WIMP-induced nuclear recoils, Ph.D. thesis, Massachusetts Institute of Technology, Cambridge, MA, 2013. URL: http://hdl . handle.net/1721.1/84395.

[44] J. P. Lopez, First results from a 20-liter prototype dark matter detector with directional sensitivity, Ph.D. thesis, Massachusetts Institute of Technology, Cambridge, MA, 2013. URL: http://academics.wellesley.edu/Physics/jbattat/dmtpc/jpl.pdf.

[45] H. Tomita, Detector development for direction-sensitive dark matter research, Ph.D. thesis, Boston University, 2011. URL: http://academics.wellesley.edu/Physics/jbattat/dmtpc/ht.pdf. 
[46] J. Lopez, K. Terao, J. Conrad, D. Dujmic, L. Winslow, A Prototype Detector for Directional Measurement of the Cosmogenic Neutron Flux, Nucl. Instrum. Meth. A673 (2012) 22-31.

[47] A. Roccaro, H. Tomita, S. Ahlen, D. Avery, A. Inglis, J. Battat, D. Dujmic, P. Fisher, S. Henderson, A. Kaboth, G. Kohse, R. Lanza, J. Monroe, G. Sciolla, N. Skvorodnev, H. Wellenstein, R. Yamamoto, A background-free direction-sensitive neutron detector, Nuclear Instruments and Methods in Physics Research A 608 (2009) 305-309.

[48] J. D. Lewin, P. F. Smith, Review of mathematics, numerical factors, and corrections for dark matter experiments based on elastic nuclear recoil, Astropart. Phys. 6 (1996) 87-112.

[49] P. C. Divari, T. S. Kosmas, J. D. Vergados, L. D. Skouras, Shell model calculations for light supersymmetric particle scattering off light nuclei, Phys. Rev. C 61 (2000) 054612.

[50] A. Kaboth, et al. (DMTPC), A measurement of photon production in electron avalanches in $\mathrm{CF}_{4}$, Nucl. Ins. Meth. Phys. Res. A592 (2008) $63-72$.

[51] A. Pansky, A. Breskin, A. Buzulutskov, R. Chechik, V. Elkind, J. Va'Vra, The scintillation of $\mathrm{CF}_{4}$ and its relevance to detection science, Nuclear Instruments and Methods in Physics Research A 354 (1995) 262-269.

[52] L. G. Christophorou, J. K. Olthoff, M. V. V. S. Rao, Electron Interac- 
tions with $\mathrm{CF}_{4}$, Journal of Physical and Chemical Reference Data 25 (1996) 1341-1388.

[53] I. Jaegle, H. Feng, S. Ross, J. Yamaoka, S. E. Vahsen, Simulation of the Directional Dark Matter Detector $\left(\mathrm{D}^{3}\right)$ and Directional Neutron Observer (DiNO), in: F. Mayet, D. Santos (Eds.), EAS Publications Series, volume 53 of EAS Publications Series, 2012, pp. 111-118. doi:10.1051/eas/1253014. arXiv:1110.3444.

[54] Loomba, D., R\&D with Implications for a Directional Low Mass Dark Matter Search, 2013.

[55] A. Morozov, M. M. F. R. Fraga, L. Pereira, L. M. S. Margato, S. T. G. Fetal, B. Guerard, G. Manzin, F. A. F. Fraga, Photon yield for ultraviolet and visible emission from $\mathrm{CF}_{4}$ excited with $\alpha$-particles, Nuclear Instruments and Methods in Physics Research B 268 (2010) 1456-1459.

[56] R. Brun, F. Rademakers, ROOT - An Object Oriented Data Analysis Framework, Nuclear Instruments and Methods in Physics Research A 389 (1997) 81-86.

[57] A. Kaboth, Detecting the invisible universe with neutrinos and dark matter, Ph.D. thesis, Massachusetts Institute of Technology, Cambridge, MA, 2012. URL: http://hdl.handle.net/1721.1/76980.

[58] T. Skwarnicki, A study of the radiative cascade transitions between the Upsilon-Prime and Upsilon resonances, Ph.D. thesis, 1986. DESY-F3186-02. 
[59] G. F. Reinking, L. G. Christophorou, S. R. Hunter, Studies of total ionization in gases/mixtures of interest to pulsed power applications, Journal of Applied Physics 60 (1986) 499-508.

[60] A. Sharma, Properties of some gas mixtures used in tracking detectors, SLAC-JOURNAL-ICFA-16-3 (1998).

[61] A. Hitachi, Bragg-like curve for dark matter searches: Binary gases, Radiation Physics and Chemistry 77 (2008) 1311-1317.

[62] I. C. Wolfe, Measurement of work function in CF4 gas, Bachelor of science thesis, Massachusetts Institute of Technology, Cambridge, MA, 2010. URL: http://hdl . handle.net/1721.1/61268.

[63] A. Bressan, M. Hoch, P. Pagano, L. Ropelewski, F. Sauli, S. Biagi, A. Buzulutskov, M. Gruwé, G. De Lentdecker, D. Moermann, A. Sharma, High rate behavior and discharge limits in micro-pattern detectors, Nuclear Instruments and Methods in Physics Research A 424 (1999) 321-342.

[64] H. Junde, Nuclear Data Sheets for A = 55, Nuclear Data Sheets 109 (2008) 787-942.

[65] NIST XCOM photon cross sections database, Accessed: 2013-11-18. URL: http://www.nist.gov/pml/data/xcom/.

[66] H. Raether, Electron avalanches and breakdown in gases, Butterworths, Washington, D.C., 1964. 
[67] D. D. Cohen, X-rays from an ${ }^{241}$ Am source and their relative intensities, Nuclear Instruments and Methods in Physics Research A 267 (1988) 492-498.

[68] J. R. Janesick, Scientific charge-coupled devices, 2001.

[69] C. Tomasi, R. Manduchi, Bilateral filtering for gray and color images, in: Computer Vision, 1998, pp. 839-846.

[70] V. Gregoric, Dark matter detection with the Dark Matter Time Projection Chamber collaboration, Master of science thesis, Bryn Mawr College, Bryn Mawr, PA, 2013. URL: http://repository.brynmawr . edu/dissertations/85/.

[71] J. F. Ziegler, M. D. Ziegler, J. P. Biersack, SRIM - The stopping and range of ions in matter (2010), Nuclear Instruments and Methods in Physics Research B 268 (2010) 1818-1823.

[72] O. Guillaudin, J. Billard, G. Bosson, O. Bourrion, T. Lamy, et al., Quenching factor measurement in low pressure gas detector for directional dark matter search, EAS Publ.Ser. 53 (2012) 119-127.

[73] T. Caldwell, et al. (DMTPC), Transport properties of electrons in $\mathrm{CF}_{4}$, arXiv/ 0905.2549 (2009).

[74] E. Behnke, J. Behnke, S. J. Brice, D. Broemmelsiek, J. I. Collar, A. Conner, P. S. Cooper, M. Crisler, C. E. Dahl, D. Fustin, E. Grace, J. Hall, M. Hu, I. Levine, W. H. Lippincott, T. Moan, T. Nania, E. Ramberg, A. E. Robinson, A. Sonnenschein, M. Szydagis, E. Vázquez-Jáuregui, 
1074

1075

1076

1077

1078

1079

1080

1081

First dark matter search results from a 4 - $\mathrm{kg} \mathrm{CF}_{3} \mathrm{I}$ bubble chamber operated in a deep underground site, Phys. Rev. D 86 (2012) 052001.

[75] M. Felizardo, T. A. Girard, T. Morlat, A. C. Fernandes, A. R. Ramos, J. G. Marques, A. Kling, J. Puibasset, M. Auguste, D. Boyer, A. Cavaillou, J. Poupeney, C. Sudre, H. S. Miley, R. F. Payne, F. P. Carvalho, M. I. Prudêncio, A. Gouveia, R. Marques, Final Analysis and Results of the Phase II SIMPLE Dark Matter Search, Phys. Rev. Lett. 108 (2012) 201302. 